\title{
SPECTRAL CLASSIFICATION AND PROPERTIES OF THE O Vz STARS IN THE GALACTIC O-STAR SPECTROSCOPIC SURVEY (GOSSS)
}

\author{
Julia I. Arias ${ }^{1}$, Nolan R. Walborn ${ }^{2}$, Sergio Simón Díaz ${ }^{3}$, Rodolfo H. Barbá ${ }^{1}$, Jesús Maíz Apellániz ${ }^{4}$, \\ Carolina Sabín-Sanjulián ${ }^{1}$, Roberto C. Gamen ${ }^{5}$, Nidia I. Morrell ${ }^{6}$, Alfredo Sota ${ }^{7}$, Amparo Marco ${ }^{8,9}$, \\ Ignacio Negueruela ${ }^{8}$, Jỗo R. S. Leão ${ }^{10}$, Artemio Herrero ${ }^{3}$, and Emilio J. Alfaro ${ }^{7}$ \\ ${ }^{1}$ Departamento de Física y Astronomía, Universidad de La Serena, Av. Cisternas 1200 Norte, La Serena, Chile; jarias@userena.cl \\ ${ }^{2}$ Space Telescope Science Institute, 3700 San Martin Drive, MD 21218, Baltimore, USA \\ ${ }^{3}$ Instituto de Astrofísica de Canarias, E-38200, Departamento de Astrofísica, Universidad de La Laguna, E-38205, La Laguna, Tenerife, Spain \\ ${ }^{4}$ Centro de Astrobiología, CSIC-INTA, campus ESAC, Camino Bajo del Castillo s/n, E-28 692 Madrid, Spain \\ 5 Instituto de Astrofísica de La Plata (CONICET, UNLP), Facultad de Ciencias Astronómicas y Geofísicas, \\ Universidad Nacional de La Plata, Paseo del Bosque s/n, 1900 La Plata, Argentina \\ ${ }^{6}$ Las Campanas Observatory, Carnegie Observatories, Casilla 601, La Serena, Chile \\ ${ }^{7}$ Instituto de Astrofísica de Andalucía-CSIC, Glorieta de la Astronomía s/n, E-18 008 Granada, Spain \\ ${ }^{8}$ Departamento de Física, Ingeniería de Sistemas y Teoría de la Señal, Escuela Politécnica Superior, Universidad de Alicante, \\ Carretera San Vicente del Raspeig s/n, E03690, San Vicente del Raspeig, Spain \\ ${ }^{9}$ Department of Astronomy, University of Florida, 211 Bryant Space Science Center, Gainesville, FL 32611, USA \\ ${ }^{10}$ Universidade Federal do Rio Grande do Norte-UFRN Caixa Postal 1524-Campus Universitrio Lagoa Nova, CEP 59078-970-Natal/RN, Brasil \\ Received 2016 January 10; accepted 2016 April 12; published 2016 July 12
}

\begin{abstract}
On the basis of the Galactic O Star Spectroscopic Survey (GOSSS), we present a detailed systematic investigation of the $\mathrm{OVz}$ stars. The currently used spectral classification criteria are rediscussed, and the Vz phenomenon is recalibrated through the addition of a quantitative criterion based on the equivalent widths of the He $\mathrm{I} \lambda 4471$, $\mathrm{He}$ II $\lambda 4542$, and $\mathrm{He}$ II $\lambda 4686$ spectral lines. The GOSSS $\mathrm{O} \mathrm{Vz}$ and $\mathrm{O} \mathrm{V}$ populations resulting from the newly adopted spectral classification criteria are comparatively analyzed. The locations of the $\mathrm{O} \mathrm{Vz}$ stars are probed, showing a concentration of the most extreme cases toward the youngest star-forming regions. The occurrence of the $\mathrm{Vz}$ spectral peculiarity in a solar-metallicity environment, as predicted by the FASTwIND code, is also investigated, confirming the importance of taking into account several processes for the correct interpretation of the phenomenon.
\end{abstract}

Key words: stars: early-type - stars: fundamental parameters - surveys

\section{INTRODUCTION}

Walborn (1973) first noted that the spectra of some O dwarfs in the very young Galactic cluster Trumpler 14 in the Carina Nebula showed substantially stronger $\mathrm{He}$ II $\lambda 4686$ absorption, relative to the other He lines, than is observed in typical class $\mathrm{V}$ spectra. After that, more extreme examples of objects showing this peculiarity were found in the Large Magellanic Cloud (LMC), in particular, in the extremely young star-forming regions N11 (Parker et al. 1992; Walborn \& Parker 1992) and 30 Doradus (Walborn \& Blades 1997). These discoveries led to the introduction, in the latter paper, of a new luminosity subclass named $\mathrm{Vz}$, where " $z$ " was chosen for the Zero Age Main Sequence (ZAMS), as this characteristic was hypothesized to be a signature of youth.

The idea behind the hypothesis that relates the Vz phenomenon to the young age of the object is that it represents an "inverse" behavior of the selective emission effect observed among $\mathrm{O}$ stars, the Of phenomenon, which is due to the emission filling of the $\mathrm{He}$ II $\lambda 4686$ line and correlates with increasing luminosity (Walborn 2001). In other words, the typical class V spectra would already have some emission filling in the He II $\lambda 4686$ line, whereas the Vz objects would have less, thus being less luminous and less evolved, i.e., they would be closer to the ZAMS.

Over the last few decades, numerous examples of objects belonging to the $\mathrm{OVz}$ class have been identified, both in the Galaxy and the Magellanic Clouds. Walborn (2009) presented a compilation of 50 optically observable ZAMS O candidates that included several stars classified as O Vz. In spite of their probable key role in understanding the early evolution of massive stars, quantitative studies of individual $\mathrm{OVz}$ stars have been very scarce for years. Moreover, some of these isolated studies place the Vz objects on, while others locate them as departing from, the ZAMS, which has provoked some controversy regarding whether or not the Vz spectroscopic classification implies extreme youth.

Very recently, the number of known O Vz stars in the LMC was significantly increased thanks to the VLT-FLAMES Tarantula Survey (VFTS) ESO Large Program (Evans et al. 2011). Its huge spectroscopic data set permitted the detection of no fewer than $48 \mathrm{O} \mathrm{Vz}$ objects among a sample of 213 O stars in the 30 Doradus region (Walborn et al. 2014). Sabín-Sanjulián et al. (2014; hereafter SS14) took advantage of this data set to perform the first quantitative analysis of a statistically meaningful sample of $\mathrm{OVz}$ stars. Using the FASTWIND stellar atmosphere code, they obtained stellar and wind parameters for $38 \mathrm{O} \mathrm{Vz}$ and $46 \mathrm{O} \mathrm{V}$ stars, and found that, in general, the $\mathrm{OVz}$ stars appear to be on or very near the ZAMS, although there are a non-negligible number of cases with more advanced ages of 2-4 Ma. They also investigated the predictions of the FASTWIND code regarding the Vz characteristic, and remarked on the fact that, in addition to effective temperature and wind strength, other stellar parameters such as gravity and projected rotational velocity must be taken into account for the correct interpretation of the phenomenon from an evolutionary point of view. An interesting conclusion of this exhaustive research concerns the role of metallicity in the $\mathrm{O} V \mathrm{z}$ phenomenon. These authors propose that the large number of $\mathrm{Vz}$ stars in 30 Doradus, and the fact that some of them are 
found away from the ZAMS, may be explained by the low metal content of the LMC, which inhibits the development of a wind strong enough to break the $\mathrm{Vz}$ characteristic. As a corollary, a lower percentage of $\mathrm{O} \mathrm{Vz}$ stars away from the ZAMS should be expected in the Galaxy.

In the Galaxy, the Galactic O Star Spectroscopic Survey (GOSSS; Maíz Apellániz et al. 2011) represents the state of the art in the spectral classification of massive hot stars. Based on high signal-to-noise ratio $(\mathrm{S} / \mathrm{N})$ observations from both hemispheres, it is the largest collection of O-star optical spectra ever assembled. Because of the quality, quantity, and homogeneity of the data, this survey has produced several systemic developments and revisions, some of them unexpected, for O-type stars. In addition, having improved the definition of the spectral classification system, GOSSS has revealed numerous objects and categories of special interest, also allowing for their statistical study (Walborn et al. 2010, 2011; Sota et al. 2011, 2014).

The present work constitutes a further example of the potential of GOSSS. Its unprecedented database has confirmed several previously known cases of stars belonging to the $\mathrm{O} \mathrm{Vz}$ class, and has led to the discovery of many new examples. Sota et al. (2011, 2014; hereafter S11 and S14, respectively) present spectral classifications for a total of 448 stars, 167 of which belong to the luminosity class V. Among these stars, 68 objects have been classified as O Vz. More than 150 additional dwarfs have been observed since the publication of the first two papers of the project, the majority of which are included in the third installment (Maíz Apellániz et al. 2016, hereafter MA16). Making use of this huge amount of new data, in this paper, we systematically investigate the properties of the $\mathrm{O}$ Vz stars in the Galaxy. This empirical analysis is also complemented by a simple theoretical study of the Vz phenomenon (Section 6). With a limited resolving power of $R \sim 2500$, the GOSSS data are not suitable for determining the stellar parameters by the use of state-of-the-art stellar atmosphere models. ${ }^{11}$ Then, following the procedure in the work by SS14, we used synthetic spectra from a grid of FASTWIND models to investigate the effect of several parameters on the occurrence of the $\mathrm{Vz}$ spectral peculiarity in a solar-metallicity environment.

\section{OBSERVATIONS}

All of the observations used in this work come from the Galactic O Star Spectroscopic Survey (GOSSS). Details on the data and analysis procedures are fully discussed in the three papers from the project (S11, S14, and MA16) and will not be repeated here. We recall only that GOSSS is a long-term systematic survey of all of the Galactic stars ever classified as $\mathrm{O}$. This project provides moderate resolution $(R \sim 2500)$ spectroscopy in the blue-violet region (approximately 3900-5000 A) with a high signal-to-noise ratio, typically $\mathrm{S} / \mathrm{N} \sim 200-300$. The spectral types are available through the latest version of the Galactic $\mathrm{O}$ Star Catalog (GOSC; Maíz Apellániz et al. 2004). In this paper, we include $226 \mathrm{O}$ stars from both hemispheres pertaining to the three published GOSSS installments.

\section{SELECTION CRITERIA FOR THE SAMPLE OF STUDY}

At the beginning of this work, GOSC contained more than 300 class V stars spanning a range of spectral types from $\mathrm{O} 3$ to 09.7 .

\footnotetext{
${ }^{11}$ In a forthcoming paper, we will present such a quantitative analysis using high-resolution spectra from two large spectroscopic surveys in the Milky Way: OWN (Barbá et al. 2010) and IACOB (Simón-Díaz et al. 2011a, 2015).
}

Spectral types for 163 of these objects had already been published by $\mathrm{S} 11$ and $\mathrm{S} 14$. We used the original spectral classifications of the latter objects as a starting point for the present study. In Figure 1, we show their spectral type distributions. The top panel shows the distribution for the 68 stars belonging at that time to the $\mathrm{Vz}$ category, whereas the bottom panel shows the distribution corresponding to the 95 objects classified as "normal" dwarfs, i.e., class V non-z. It must be stressed that these spectral classifications were obtained prior to the introduction of the quantitative criterion defined in this paper. ${ }^{12}$ The overall picture was the same as observed for the dwarfs in the 30 Doradus region (see Figure 1 in SS14): the O Vz stars showed a marked concentration toward the intermediate spectral type $\mathrm{O} 7$, with a complete lack of objects at spectral types later than O8.5, whereas the bulk of the normal dwarfs presented spectral types later than O8.

The observed lack of late-type objects among the $\mathrm{O} V \mathrm{z}$ stars was not surprising since, strictly speaking, stars with spectral types later than $\mathrm{O} 9$ are not relevant to the $\mathrm{Vz}$ phenomenon. As was clearly demonstrated by SS14, the Vz spectral characteristic will never be observed at the effective temperatures typical of these objects. Based on models computed for the metallicity of the LMC, these authors showed that below a certain value of $T_{\text {eff }}$, the natural behavior of the He lines is such that $\mathrm{He} I \lambda 4471$ is always stronger in absorption than He II $\lambda 4686$, independently of the wind strength. In Section 6, we will demonstrate that a similar argument is also valid in the Galaxy. Consequently, all of the stars with spectral types later than or equal to $\mathrm{O} 9$ were not taken into account in the present analysis of the $\mathrm{O}$ Vz class. It is interesting to note that if we restrict the GOSSS published sample to the O3O8.5 range, then the total numbers of objects found at that moment for the $\mathrm{Vz}$ and $\mathrm{V}$ non- $z$ categories were surprisingly similar (68 and 61, respectively). This means that the $\mathrm{O} \mathrm{Vz}$ objects constituted $\sim 52 \%$ of the class $\mathrm{V}$ stars in the relevant spectral type range.

Considering the previous constraint regarding spectral types, our sample of interest decreased to 223 class V stars in the spectral type range O3-O8.5. These objects have been subdivided into different categories according to the following criteria. As this study involves the measurements of the equivalent widths (EWs) and central depths (CDs) of the lines present in the GOSSS spectra, we used the appearance of the spectral lines at the relatively low resolution of GOSSS, along with the available binarity information, in order to separate them into different groups. We will go deeply into the impact of binaries in Section 4.4, but suffice it to say here that they may, for example, produce false $\mathrm{O} \mathrm{Vz}$ spectra, and hence we had to be extremely careful when probing the binary nature of our sample objects. In addition to the multiple-epoch GOSSS spectra, information from the literature and/or high-resolution surveys was used to identify as many binaries as possible. Thus, the categories and numbers that characterize our sample objects are the following: (1) objects that are single lined in the GOSSS spectra and for which no evidence of binarity is known (132 stars listed in Table 1); (2) objects that are single lined in the GOSSS spectra but are known to be spectroscopic binaries (SBs) from high-resolution data (45 binaries, Table 2); (3) objects that are double lined in the GOSSS spectra (explicit SB2) for which the line separation is sufficiently large to allow measurements of the

\footnotetext{
12 In MA16, the Vz classification scheme was adapted to the results presented in this paper; previous spectral classifications were thus revised and modified accordingly.
} 

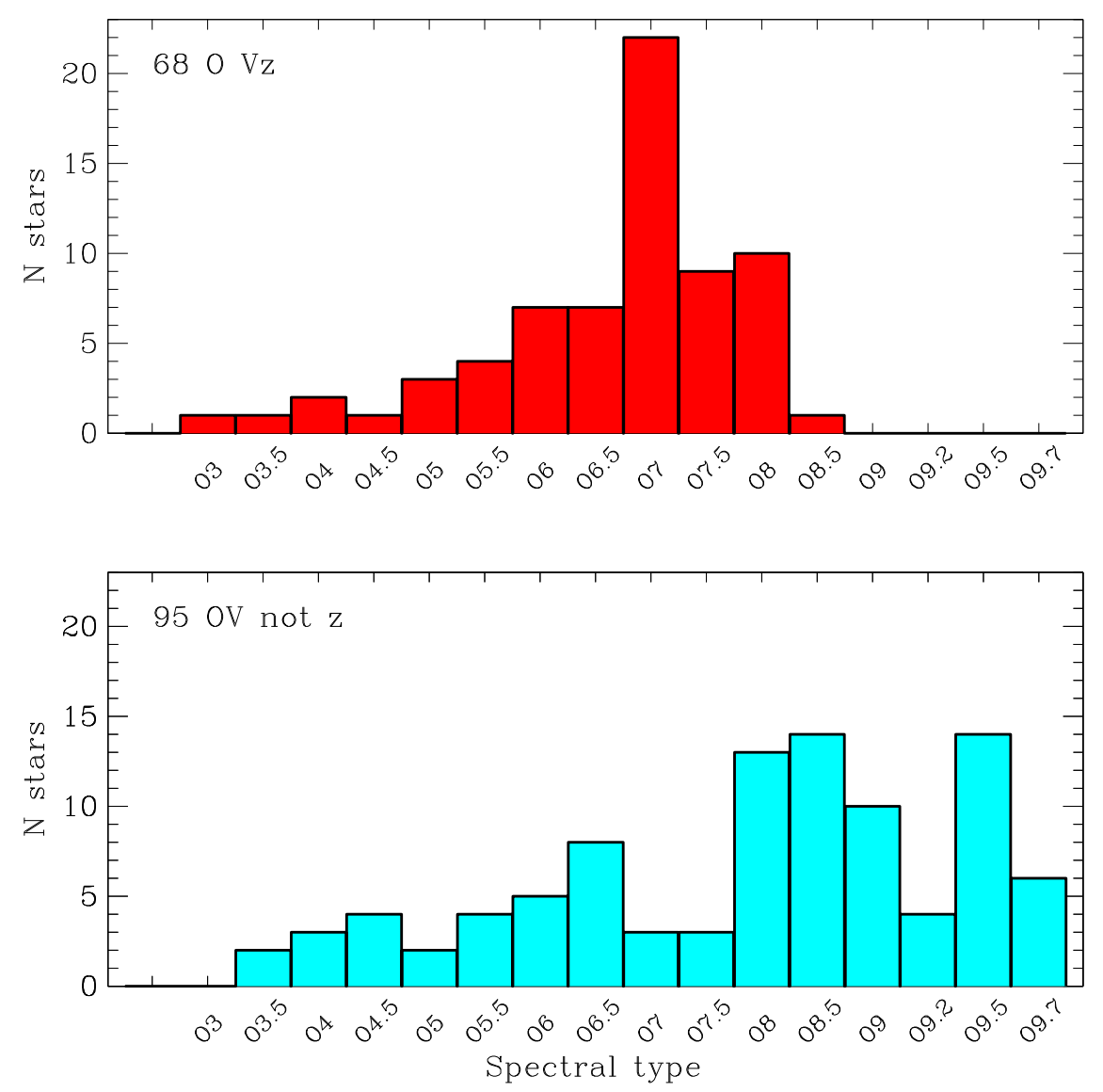

Figure 1. Number of stars ( $\mathrm{N}$ stars) as a function of spectral type for the Galactic $\mathrm{O} \mathrm{Vz}$ (top) and $\mathrm{O} \mathrm{V}$ non- $z$ stars (bottom) included in the first two papers of the GOSSS project.

CDs and EWs of the individual components by the use of deblending methods (23 binaries providing 32 components with spectral types earlier than O9); and (4) explicit SB2 whose spectral components are not sufficiently separated to be measured individually (15 binaries). Binaries belonging to groups (3) and (4) are listed in Table 3. A fifth category includes those objects with peculiar spectral characteristics, for example, emission line spectra, magnetic stars, and interacting binaries (8 stars). In the following sections, the role and significance of each of the former categories will be explained in detail.

\section{SPECTRAL CLASSIFICATION CRITERIA FOR THE O VZ SUBCLASS}

\subsection{On the Problems with the CD-based Classifications}

Specifically, the current spectroscopic criterion for assigning the luminosity class $\mathrm{Vz}$ to $\mathrm{O}$ stars is that $\mathrm{He}$ II $\lambda 4686$ absorption is stronger than any other He line at every spectral type, i.e., it is stronger than He II $\lambda 4542$ at types earlier than or equal to O6.5, stronger than $\mathrm{He}_{\mathrm{I}} \lambda 4471$ at types later than or equal to $\mathrm{O} 7.5$, and stronger than both at type $\mathrm{O} 7$ where they are equal by definition.

In the context of the morphological spectral classification scheme, from the advent of the digital data, the former criterion is generally applied by comparing the central depths (CDs) of the involved lines. However, it is well known that the appearances of the spectral lines may be highly modified by many effects, and that the amount and characteristics of these changes depend on the specific line observed. For example, rotational broadening alters the depths of the $\mathrm{He}_{\mathrm{I}}$ and $\mathrm{He}$ II lines differently due to the different intrinsic widths of these lines caused by the Stark effect, which is linear in He II and quadratic in He I. In particular, the effects of rotation on the morphologically derived spectral types are discussed by Markova et al. (2011). Similar effects obviously may affect the $\mathrm{Vz}$ morphological spectral classification. Regarding this point, using FASTWIND models computed for $Z=0.5 \mathrm{Z}_{\odot}$ (corresponding to the metallicity of the LMC), SS14 studied the role of rotational broadening in the $\mathrm{Vz}$ phenomenon, finding that if we define the $\mathrm{Vz}$ characteristic from the relative depths of the He lines, then higher values of $v \sin i$ generally favor the inference of $\mathrm{O} \mathrm{Vz}$ classifications at relatively low temperatures, with the opposite effect in the high temperature regime.

We explored the former effect using FASTwIND (SantolayaRey et al. 1997; Puls et al. 2005) models computed for a metallicity value of $Z=Z_{\odot}$, which is reasonable for the Galactic stars studied here, and obtained a similar result. A convenient grid of models was chosen among the various grids included in the IACOB-Grid Based Automatic Tool (IACOBGBAT; Simón-Díaz et al. 2011b). In Figure 2, the ratio between the CDs of the He II 4686 and He I 4471 absorptions is plotted against the effective temperature for three values of the projected rotational velocity, mantaining fixed the resolution, the surface gravity, and the wind-strength parameter $Q .{ }^{13} \mathrm{We}$ note, for example, that the spectrum of a star whose effective

\footnotetext{
13 The wind-strength parameter $Q$ relates the mass-loss rate $\dot{M}$, the terminal velocity $v_{\infty}$, and the stellar radius $R$, under the optical-depth invariant $Q=\dot{M}\left(R v_{\infty}\right)^{-3 / 2}$.
} 
Table 1

O V Stars in GOSSS with No Evidence of Binarity

\begin{tabular}{|c|c|c|c|c|c|c|c|c|}
\hline Object & $\begin{array}{l}\text { R.A. } \\
\text { hh:mm: } \\
\text { ss.ss }\end{array}$ & $\begin{array}{c}\text { decl. } \\
\text { dd:am:as.s }\end{array}$ & Sp. Type & References & EW(He I 4471) & EW(He II 4542) & $\begin{array}{c}\text { EW(He II 4686) } \\
(\AA)\end{array}$ & $z$ \\
\hline $\mathrm{BD}+661675$ & 00:02:10.29 & $+67: 24: 32.2$ & $07.5 \mathrm{Vz}$ & MA16 & 0.70 & 0.68 & 0.88 & 1.25 \\
\hline Тус 4026-00424-1 & 00:02:19.03 & $+67: 25: 38.5$ & $07 \mathrm{~V}((\mathrm{f})) \mathrm{z}$ & MA16 & 0.55 & 0.66 & 0.84 & 1.27 \\
\hline ALS 6351 & $00: 48: 12.55$ & $+62: 59: 24.8$ & $\mathrm{O} 7 \mathrm{Vz}$ & MA16 & 0.71 & 0.90 & 1.04 & 1.16 \\
\hline HD 5005A & $00: 52: 49.21$ & $+56: 37: 39.5$ & $\mathrm{O} 4 \mathrm{~V}((\mathrm{fc}))$ & S11a & 0.19 & 0.76 & 0.81 & 1.07 \\
\hline HD 5005C & $00: 52: 49.55$ & $+56: 37: 36.8$ & $\mathrm{O} 8.5 \mathrm{~V}(\mathrm{n})$ & S11a & 0.85 & 0.81 & 0.76 & 0.89 \\
\hline $\mathrm{BD}+60134$ & $00: 56: 14.22$ & $+61: 45: 36.9$ & $05.5 \mathrm{~V}(\mathrm{n})((\mathrm{f}))$ & MA16 & 0.50 & 0.91 & 1.00 & 1.10 \\
\hline HD 5689 & $00: 59: 47.59$ & $+63: 36: 28.3$ & O7 Vn ((f)) & MA16 & 0.70 & 0.92 & 0.71 & 0.78 \\
\hline HD 12993 & 02:09:02.47 & $+57: 55: 55.9$ & O6.5 V((f)) Nstr & $\mathrm{S} 14$ & 0.70 & 0.95 & 1.00 & 1.05 \\
\hline $\mathrm{BD}+61411 \mathrm{~A}$ & 02:26:34.39 & $+62: 00: 42.4$ & O6.5 V((f))z & MA16 & 0.46 & 0.73 & 0.89 & 1.22 \\
\hline $\mathrm{BD}+60501$ & $02: 32: 36.27$ & $+61: 28: 25.6$ & $\mathrm{O} 7 \mathrm{~V}(\mathrm{n})((\mathrm{f})) \mathrm{z}$ & S11a & 0.57 & 0.75 & 0.91 & 1.21 \\
\hline HD 15629 & $02: 33: 20.59$ & $+61: 31: 18.2$ & $\mathrm{O} 4.5 \mathrm{~V}((\mathrm{fc}))$ & S11a & 0.20 & 0.66 & 0.52 & 0.78 \\
\hline $\mathrm{BD}+60513$ & 02:34:02.53 & $+61: 23: 10.9$ & O7 Vn & MA16 & 0.61 & 0.82 & 0.75 & 0.91 \\
\hline $\mathrm{BD}+62424$ & $02: 36: 18.22$ & $+62: 56: 53.4$ & O6.5 V(n)((f)) & S11a & 0.45 & 0.80 & 0.73 & 0.91 \\
\hline HD $17505 B$ & 02:51:08.26 & $+60: 25: 03.8$ & $\mathrm{O} 8 \mathrm{~V}$ & S11a & 0.70 & 0.54 & 0.69 & 0.99 \\
\hline $\mathrm{BD}+60586 \mathrm{~A}$ & $02: 54: 10.67$ & $+60: 39: 03.6$ & $07 \mathrm{Vz}$ & S11a & 0.68 & 0.67 & 0.83 & 1.23 \\
\hline ALS 7833 & 03:59:07.49 & $+57: 14: 11.7$ & $\mathrm{O} 8 \mathrm{Vz}$ & MA16 & 0.72 & 0.70 & 0.87 & 1.20 \\
\hline $\mathrm{BD}+50886$ & 04:03:20.74 & $+51: 18: 52.5$ & $\mathrm{O} 4 \mathrm{~V}((\mathrm{fc}))$ & MA16 & 0.12 & 0.74 & 0.79 & 1.07 \\
\hline $\mathrm{BD}+52805$ & 04:18:35.64 & $+52: 51: 54.3$ & O8 V(n) & MA16 & 0.80 & 0.77 & 0.86 & 1.07 \\
\hline ALS 8294 & $05: 22: 39.69$ & $+33: 22: 18.2$ & $\mathrm{O} 7 \mathrm{~V}(\mathrm{n}) \mathrm{z}$ & MA16 & 0.83 & 0.81 & 0.93 & 1.12 \\
\hline HDE 242926 & $05: 22: 40.10$ & $+33: 19: 09.4$ & $\mathrm{O} 7 \mathrm{Vz}$ & S11 & 0.61 & 0.77 & 0.88 & 1.14 \\
\hline $\mathrm{BD}+331025 \mathrm{~A}$ & $05: 22: 44.00$ & $+33: 26: 26.6$ & $07.5 \mathrm{~V}(\mathrm{n}) \mathrm{z}$ & $\mathrm{S} 14$ & 0.81 & 0.87 & 0.96 & 1.11 \\
\hline HDE 242935 A & $05: 22: 46.54$ & $+33: 25: 11.5$ & O6.5 V((f))z & S11a & 0.49 & 0.83 & 0.92 & 1.12 \\
\hline HD 35619 & $05: 27: 36.15$ & $+34: 45: 19.0$ & O7.5 V((f)) & MA16 & 0.73 & 0.64 & 0.71 & 0.97 \\
\hline HD 36879 & $05: 35: 40.53$ & $+21: 24: 11.7$ & $\mathrm{O} 7 \mathrm{~V}(\mathrm{n})((\mathrm{f}))$ & MA16 & 0.60 & 0.76 & 0.72 & 0.95 \\
\hline HD 41161 & 06:05:52.46 & $+48: 14: 57.4$ & O8 Vn & S11 & 0.98 & 0.87 & 0.78 & 0.80 \\
\hline HD 41997 & 06:08:55.82 & $+15: 42: 18.2$ & O7.5 Vn((f)) & S11 & 0.71 & 0.77 & 0.55 & 0.72 \\
\hline HD 42088 & 06:09:39.57 & $+20: 29: 15.5$ & O6 V((f))z & S11 & 0.63 & 0.81 & 1.06 & 1.30 \\
\hline HD 44811 & $06: 24: 38.35$ & $+19: 42: 15.8$ & $\mathrm{O} 7 \mathrm{~V}(\mathrm{n}) \mathrm{z}$ & $\mathrm{S} 11$ & 0.70 & 0.75 & 0.95 & 1.27 \\
\hline ALS 19265 & $06: 24: 59.85$ & $+26: 49: 20.0$ & $\mathrm{O} 4.5 \mathrm{~V}((\mathrm{c})) \mathrm{z}$ & MA16 & 0.31 & 1.10 & 1.24 & 1.13 \\
\hline HDE 256725 A & $06: 25: 01.30$ & $+19: 50: 56.1$ & O5 V((fc)) & MA16 & 0.34 & 0.94 & 0.92 & 0.97 \\
\hline HD 46056 A & $06: 31: 20.86$ & $+04: 50: 03.9$ & O8 Vn & S11 & 0.80 & 0.69 & 0.75 & 0.94 \\
\hline HD 46150 & $06: 31: 55.52$ & $+04: 56: 34.3$ & O5 V((f))z & S11 & 0.26 & 0.57 & 0.79 & 1.39 \\
\hline HD 46573 & 06:32:09.31 & $+04: 49: 24.7$ & $\mathrm{O} 4 \mathrm{~V}((\mathrm{f}))$ & $\mathrm{S} 11$ & 0.16 & 0.70 & 0.70 & 0.99 \\
\hline HD 46485 & $06: 33: 50.96$ & $+04: 31: 31.6$ & O7 V((f))n var? & MA16 & 0.80 & 0.78 & 0.82 & 1.03 \\
\hline Тус 0737-01170-1 & $06: 36: 29.00$ & $+10: 49: 20.7$ & $\mathrm{O} 7 \mathrm{Vz}$ & MA16 & 0.61 & 0.77 & 0.98 & 1.27 \\
\hline HD 48279 A & $06: 42: 40.55$ & $+01: 42: 58.2$ & O8.5 V Nstr var? & MA16 & 0.98 & 0.90 & 0.96 & 0.98 \\
\hline ALS 207 & 07:09:55.20 & $-18: 30: 07.9$ & O6.5 V((f)) & MA16 & 0.57 & 0.91 & 0.98 & 1.08 \\
\hline HD 57236 & 07:19:30.10 & $-22: 00: 17.3$ & $08.5 \mathrm{~V}$ & S14 & 0.83 & 0.59 & 0.85 & 1.03 \\
\hline ALS 458 & 07:30:01.27 & $-19: 08: 35.0$ & O6.5 V((f))z & MA16 & 0.59 & 0.80 & 0.91 & 1.14 \\
\hline BD -15 1909 & $07: 34: 58.46$ & $-16: 14: 23.2$ & O6.5 V((f))z & MA16 & 0.61 & 0.81 & 0.93 & 1.15 \\
\hline CPD -26 2704 & $07: 52: 36.52$ & $-26: 22: 42.0$ & O7 V(n) & MA16 & 0.78 & 0.89 & 0.88 & 0.99 \\
\hline HD 64568 & $07: 53: 38.21$ & $-26: 14: 02.6$ & O3 V $\left(\left(f^{*}\right)\right) z$ & S14 & 0.00 & 0.78 & 0.95 & 1.23 \\
\hline CPD -47 2962 & 08:57:51.66 & $-47: 45: 43.9$ & O7 V((f)) & MA16 & 0.64 & 0.95 & 0.86 & 0.91 \\
\hline CPD -49 2322 & $09: 15: 52.79$ & $-50: 00: 43.8$ & O7.5 V((f)) & MA16 & 0.69 & 0.56 & 0.68 & 0.99 \\
\hline HDE 298429 & $09: 30: 37.25$ & $-51: 39: 34.7$ & $08.5 \mathrm{~V}$ & S14 & 0.92 & 0.60 & 0.73 & 0.80 \\
\hline 2MASS J10224096-5930305 & 10:22:40.96 & $-59: 30: 30.6$ & $\mathrm{O} 7 \mathrm{~V}((\mathrm{f})) \mathrm{z}$ & MA16 & 0.55 & 0.58 & 0.74 & 1.27 \\
\hline 2MASS J10224377-5930182 & $10: 22: 43.77$ & $-59: 30: 18.2$ & O8 V(n) & MA16 & 0.75 & 0.50 & 0.61 & 0.81 \\
\hline HD 90273 & $10: 23: 44.45$ & $-57: 38: 31.5$ & ON7 V((f)) & MA16 & 0.68 & 0.85 & 0.87 & 1.03 \\
\hline HD 91837 & $10: 34: 49.51$ & $-60: 11: 14.1$ & O8.5 Vn & S14 & 0.84 & 0.62 & 0.72 & 0.86 \\
\hline HD $92206+B$ & $10: 37: 22.96$ & $-58: 37: 23.0$ & O6 V((f)) & MA16 & 0.48 & 0.75 & 0.81 & 1.08 \\
\hline HD 92504 & $10: 39: 36.87$ & $-57: 27: 40.7$ & $\mathrm{O} 8.5 \mathrm{~V}(\mathrm{n})$ & S14 & 0.83 & 0.58 & 0.80 & 0.96 \\
\hline HDE 305438 & $10: 42: 43.77$ & $-59: 54: 16.5$ & $\mathrm{O} 8 \mathrm{Vz}$ & S14 & 0.73 & 0.89 & 0.98 & 1.10 \\
\hline HDE 303316 A & $10: 43: 11.18$ & $-59: 44: 21.0$ & $\mathrm{O} 7 \mathrm{~V}((\mathrm{f})) \mathrm{z}$ & $\mathrm{S} 14$ & 0.52 & 0.64 & 0.90 & 1.42 \\
\hline CPD -58 2611 & 10:43:46.69 & $-59: 32: 54.8$ & O6 V((f))z & $\mathrm{S} 14$ & 0.45 & 0.71 & 0.92 & 1.29 \\
\hline HD 93128 & 10:43:54.37 & $-59: 32: 57.4$ & $\mathrm{O} 3.5 \mathrm{~V}((\mathrm{fc})) \mathrm{z}$ & S14 & 0.08 & 0.68 & 0.79 & 1.16 \\
\hline Trumpler 14-9 & $10: 43: 55.35$ & $-59: 32: 48.6$ & $08.5 \mathrm{~V}$ & S14 & 0.85 & 0.67 & 0.87 & 1.03 \\
\hline HD $93129 \mathrm{~B}$ & $10: 43: 57.64$ & $-59: 32: 53.5$ & $\mathrm{O} 3.5 \mathrm{~V}((\mathrm{f})) \mathrm{z}$ & MA16 & 0.13 & 0.74 & 0.83 & 1.12 \\
\hline CPD -58 2620 & 10:43:59.92 & $-59: 32: 25.4$ & O7 V((f))z & S14 & 0.59 & 0.66 & 0.96 & 1.46 \\
\hline HD 93204 & $10: 44: 32.34$ & $-59: 44: 31.0$ & O5.5 V((f)) & S14 & 0.33 & 0.81 & 0.65 & 0.80 \\
\hline HDE 303311 & $10: 44: 37.46$ & $-59: 32: 55.4$ & O6 V((f))z & $\mathrm{S} 14$ & 0.41 & 0.78 & 1.02 & 1.31 \\
\hline HDE 305524 & $10: 44: 45.24$ & $-59: 54: 41.6$ & O6.5 $\mathrm{Vn}((\mathrm{f})) \mathrm{z}$ & S14 & 0.57 & 0.67 & 0.83 & 1.23 \\
\hline CPD -59 2610 & $10: 44: 54.71$ & $-59: 56: 01.9$ & $08.5 \mathrm{~V}$ & S14 & 0.81 & 0.78 & 0.84 & 1.04 \\
\hline
\end{tabular}


Table 1

(Continued)

\begin{tabular}{|c|c|c|c|c|c|c|c|c|}
\hline Object & $\begin{array}{l}\text { R.A. } \\
\text { hh:mm: } \\
\text { ss.ss }\end{array}$ & $\begin{array}{c}\text { decl. } \\
\text { dd:am:as.s }\end{array}$ & Sp. Type & References & $\begin{array}{c}\mathrm{EW}(\mathrm{He} \text { I } 4471) \\
(\AA)\end{array}$ & $\begin{array}{c}\text { EW(He II 4542) } \\
(\AA)\end{array}$ & $\begin{array}{c}\text { EW(He II 4686) } \\
\text { (̊) }\end{array}$ & $z$ \\
\hline [ARV2008] 206 & $10: 45: 22.28$ & $-59: 50: 47.1$ & O6 V((f)) & S14 & 0.40 & 0.68 & 0.66 & $\overline{0.98}$ \\
\hline HDE 305532 & $10: 45: 34.07$ & $-59: 57: 26.7$ & $06.5 \mathrm{~V}((\mathrm{f})) \mathrm{z}$ & S14 & 0.49 & 0.72 & 0.95 & 1.32 \\
\hline CPD -59 2673 & $10: 46: 22.46$ & $-59: 53: 20.5$ & $05.5 \mathrm{~V}(\mathrm{n})((\mathrm{f})) \mathrm{z}$ & S14 & 0.40 & 0.76 & 0.92 & 1.21 \\
\hline HDE 305539 & $10: 46: 33.07$ & $-60: 04: 12.6$ & $\mathrm{O} 8 \mathrm{Vz}$ & S14 & 0.79 & 0.73 & 0.89 & 1.13 \\
\hline HDE 305612 & $10: 47: 16.42$ & $-60: 05: 40.0$ & O8 V(n)z & S14 & 0.75 & 0.68 & 0.83 & 1.11 \\
\hline HD 96715 & $11: 07: 32.82$ & $-59: 57: 48.7$ & $\mathrm{O} 4 \mathrm{~V}((\mathrm{f})) \mathrm{z}$ & S14 & 0.21 & 0.67 & 0.94 & 1.40 \\
\hline HD 97848 & $11: 14: 31.90$ & $-59: 01: 28.8$ & $\mathrm{O} 8 \mathrm{~V}$ & S14 & 0.82 & 0.67 & 0.76 & 0.92 \\
\hline NGC $3603 H S T-51$ & $11: 15: 07.50$ & $-61: 15: 46.3$ & O5.5 V(n) & MA16 & 0.22 & 0.64 & 0.52 & 0.81 \\
\hline NGC 3603 MTT 25 & $11: 15: 11.32$ & $-61: 15: 55.6$ & O5 V(n) & MA16 & 0.23 & 0.66 & 0.49 & 0.74 \\
\hline HD 97966 & $11: 15: 11.78$ & $-59: 24: 58.3$ & $\mathrm{O} 7 \mathrm{~V}((\mathrm{f})) \mathrm{z}$ & MA16 & 0.54 & 0.61 & 0.86 & 1.40 \\
\hline HD 99546 & $11: 26: 36.90$ & $-59: 26: 13.6$ & O7.5 V((f)) Nstr & MA16 & 0.72 & 0.82 & 0.78 & 0.95 \\
\hline HD 101223 & $11: 38: 22.77$ & $-63: 12: 02.8$ & $\mathrm{O} 8 \mathrm{~V}$ & S14 & 0.78 & 0.51 & 0.72 & 0.92 \\
\hline HD 110360 & $12: 42: 12.70$ & $-60: 39: 08.7$ & ON7 V & MA16 & 0.74 & 1.02 & 1.06 & 1.04 \\
\hline CPD -613973 & $13: 45: 21.10$ & $-62: 25: 35.4$ & $\mathrm{O} 7.5 \mathrm{~V}((\mathrm{f}))$ & MA16 & 0.76 & 0.79 & 0.84 & 1.06 \\
\hline HD 122313 & 14:03:12.99 & $-62: 15: 38.6$ & $08.5 \mathrm{~V}$ & MA16 & 0.91 & 0.56 & 0.85 & 0.93 \\
\hline HD 144647 & $16: 09: 16.20$ & $-49: 36: 21.8$ & $\mathrm{O} 8.5 \mathrm{~V}(\mathrm{n})$ & MA16 & 0.88 & 0.63 & 0.81 & 0.91 \\
\hline HDE $329100 \mathrm{~A}$ & $16: 54: 42.30$ & $-45: 15: 14.8$ & $\mathrm{O} 8 \mathrm{~V}(\mathrm{n})$ & MA16 & 0.79 & 0.62 & 0.64 & 0.81 \\
\hline HDE 326775 & $17: 05: 31.31$ & $-41: 31: 20.1$ & O6.5V(n)((f))z & MA16 & 0.48 & 0.69 & 0.80 & 1.16 \\
\hline ALS 18770 & 17:19:00.80 & $-38: 49: 23.1$ & $\mathrm{O} 7 \mathrm{~V}((\mathrm{f}))$ & MA16 & 0.55 & 0.75 & 0.76 & 1.01 \\
\hline ALS 18768 & 17:19:01.05 & $-38: 48: 58.9$ & $08.5 \mathrm{~V}$ & S14 & 1.03 & 0.52 & 1.00 & 0.97 \\
\hline Pismis $24-15$ & $17: 24: 28.95$ & $-34: 14: 50.7$ & $07.5 \mathrm{Vz}$ & MA16 & 0.83 & 0.66 & 0.93 & 1.12 \\
\hline ALS 17696 & $17: 24: 42.33$ & $-34: 13: 21.4$ & O7.5: V & $\mathrm{S} 14$ & 0.76 & 1.13 & 0.87 & 0.77 \\
\hline Pismis $24-2$ & $17: 24: 43.31$ & $-34: 12: 44.2$ & O5 V((f)) & S14 & 0.31 & 0.74 & 0.80 & 1.08 \\
\hline ALS 16052 & $17: 24: 45.78$ & $-34: 09: 39.9$ & O6 V((f))z & S14 & 0.48 & 0.65 & 0.87 & 1.34 \\
\hline HD 164492 A & $18: 02: 23.55$ & $-23: 01: 51.1$ & $07.5 \mathrm{Vz}$ & S14 & 0.66 & 0.82 & 1.05 & 1.27 \\
\hline ALS 4626 & 18:04:17.88 & $-13: 06: 13.7$ & ON6 V((f)) & MA16 & 0.50 & 1.13 & 1.11 & 0.98 \\
\hline HD 167633 & $18: 16: 49.66$ & $-16: 31: 04.3$ & $06.5 \mathrm{~V}((\mathrm{f}))$ & $\mathrm{S} 11$ & 0.48 & 0.86 & 0.80 & 0.93 \\
\hline ALS 4880 & $18: 17: 33.67$ & $-12: 05: 42.8$ & O6 V((f)) & MA16 & 0.47 & 0.72 & 0.76 & 1.05 \\
\hline ALS 15360 & $18: 18: 37.48$ & $-13: 43: 39.2$ & $\mathrm{O} 7 \mathrm{~V}((\mathrm{f})) \mathrm{z}$ & MA16 & 0.50 & 0.80 & 0.90 & 1.13 \\
\hline HD 168461 & 18:20:17.18 & $-12: 10: 19.2$ & O7.5 V((f))Nstr & MA16 & 0.72 & 0.84 & 0.77 & 0.92 \\
\hline HD 168504 & $18: 20: 34.10$ & $-13: 57: 15.7$ & $\mathrm{O} 7.5 \mathrm{~V}(\mathrm{n}) \mathrm{z}$ & MA16 & 0.71 & 0.75 & 0.88 & 1.18 \\
\hline ALS 19618 & $18: 20: 34.50$ & $-16: 10: 11.7$ & $\mathrm{O} 4 \mathrm{~V}(\mathrm{n})((\mathrm{fc}))$ & MA16 & 0.18 & 0.78 & 0.75 & 0.96 \\
\hline BD -14 5014 & $18: 22: 22.31$ & $-14: 37: 08.5$ & $\mathrm{O} 7.5 \mathrm{~V}(\mathrm{n})((\mathrm{f}))$ & MA16 & 0.55 & 0.50 & 0.58 & 1.06 \\
\hline $\mathrm{BD}-104682$ & $18: 24: 20.65$ & $-10: 48: 34.3$ & $\mathrm{O} 7 \mathrm{Vn}((\mathrm{f}))$ & MA16 & 0.66 & 0.90 & 0.82 & 0.90 \\
\hline $\mathrm{BD}-145040$ & $18: 25: 38.90$ & $-14: 45: 05.7$ & $05.5 \mathrm{~V}(\mathrm{n})((\mathrm{f}))$ & MA16 & 0.38 & 0.77 & 0.83 & 1.07 \\
\hline $\mathrm{BD}-044503$ & $18: 35: 32.54$ & $-04: 47: 55.4$ & $07 \mathrm{~V}$ & MA16 & 0.79 & 0.95 & 0.92 & 0.97 \\
\hline HDE 344758 & $19: 41: 52.72$ & $+24: 20: 51.1$ & $08.5 \mathrm{~V}(\mathrm{n})((\mathrm{f}))$ & MA16 & 0.83 & 0.69 & 0.70 & 0.84 \\
\hline HDE 344777 & $19: 42: 11.47$ & $+23: 26: 00.5$ & $07.5 \mathrm{Vz}$ & MA16 & 0.69 & 0.70 & 0.79 & 1.12 \\
\hline HDE 344784 A & $19: 43: 10.97$ & $+23: 17: 45.4$ & $06.5 \mathrm{~V}((\mathrm{f})) \mathrm{z}$ & MA16 & 0.48 & 0.58 & 0.69 & 1.18 \\
\hline HDE 338916 & $19: 45: 42.12$ & $+25: 21: 16.4$ & $07.5 \mathrm{Vz}$ & MA16 & 0.73 & 0.84 & 0.95 & 1.13 \\
\hline HDE 227018 & $19: 59: 49.10$ & $+35: 18: 33.5$ & O6.5 V((f))z & MA16 & 0.47 & 0.69 & 0.87 & 1.26 \\
\hline HDE 227245 & 20:02:21.71 & $+35: 40: 29.8$ & $\mathrm{O} 7 \mathrm{~V}((\mathrm{f})) \mathrm{z}$ & MA16 & 0.56 & 0.66 & 0.87 & 1.32 \\
\hline HDE 227465 & $20: 04: 27.22$ & $+33: 42: 18.4$ & O7 V((f)) & MA16 & 0.66 & 0.68 & 0.64 & 0.94 \\
\hline HD 191978 & $20: 10: 58.28$ & $+41: 21: 09.9$ & $08 \mathrm{~V}$ & MA16 & 0.80 & 0.54 & 0.82 & 1.03 \\
\hline HDE 228759 & $20: 17: 07.54$ & $+41: 57: 26.5$ & O6.5V(n)((f))z & MA16 & 0.53 & 0.67 & 0.80 & 1.20 \\
\hline ALS 18707 & $20: 17: 41.93$ & $+36: 45: 25.6$ & $06.5 \mathrm{~V}((\mathrm{f})) \mathrm{z}$ & MA16 & 0.44 & 0.70 & 0.79 & 1.13 \\
\hline HDE 228841 & $20: 18: 29.69$ & $+38: 52: 39.8$ & O6.5 $\operatorname{Vn}((\mathrm{f}))$ & $\mathrm{S} 11$ & 0.60 & 0.89 & 0.83 & 0.93 \\
\hline HD 193595 & $20: 19: 31.33$ & $+39: 03: 26.2$ & $\mathrm{O} 7 \mathrm{~V}((\mathrm{f}))$ & MA16 & 0.66 & 0.92 & 0.99 & 1.08 \\
\hline HDE 229202 & $20: 23: 22.84$ & $+40: 09: 22.5$ & $\mathrm{O} 7.5 \mathrm{~V}(\mathrm{n})((\mathrm{f}))$ & MA16 & 0.65 & 0.64 & 0.66 & 1.01 \\
\hline ALS 11355 & $20: 27: 17.57$ & $+39: 44: 32.6$ & O8 V(n)((f)) & MA16 & 0.75 & 0.59 & 0.71 & 0.95 \\
\hline $\mathrm{BD}+404179$ & $20: 27: 43.62$ & $+40: 35: 43.5$ & $\mathrm{O} 8 \mathrm{Vz}$ & MA16 & 0.70 & 0.72 & 0.81 & 1.12 \\
\hline 2MASS J20315961+4114504 & $20: 31: 59.61$ & $+41: 14: 50.5$ & $07.5 \mathrm{Vz}$ & MA16 & 0.62 & 0.81 & 0.96 & 1.18 \\
\hline Cyg OB2-6 & $20: 32: 45.45$ & $+41: 25: 37.5$ & $\mathrm{O} 8.5 \mathrm{~V}(\mathrm{n})$ & MA16 & 0.79 & 0.61 & 0.71 & 0.91 \\
\hline ALS 15111 & $20: 32: 59.13$ & $+41: 24: 25.0$ & $\mathrm{O} 8 \mathrm{~V}$ & MA16 & 0.82 & 0.59 & 0.83 & 1.01 \\
\hline Cyg OB2-22 B & $20: 33: 08.83$ & $+41: 13: 17.4$ & O6 V((f)) & S11 & 0.43 & 0.66 & 0.56 & 0.85 \\
\hline Cyg OB2-8 D & $20: 33: 16.33$ & $+41: 19: 02.0$ & $\mathrm{O} 8.5 \mathrm{~V}(\mathrm{n})$ & S14 & 0.75 & 0.46 & 0.76 & 1.02 \\
\hline Cyg OB2-24 & $20: 33: 17.48$ & $+41: 17: 09.3$ & $\mathrm{O} 8 \mathrm{~V}(\mathrm{n})$ & S11 & 0.71 & 0.74 & 0.68 & 0.92 \\
\hline Cyg OB2-25 A & $20: 33: 25.54$ & $+41: 33: 26.7$ & $\mathrm{O} 8 \mathrm{Vz}$ & MA16 & 0.69 & 0.59 & 0.82 & 1.19 \\
\hline ALS 15134 & $20: 33: 26.76$ & $+41: 10: 59.5$ & $\mathrm{O} 8 \mathrm{Vz}$ & MA16 & 0.76 & 0.67 & 0.87 & 1.15 \\
\hline $\mathrm{BD}+453216 \mathrm{~A}$ & $20: 33: 50.37$ & $+45: 39: 40.9$ & O5 V((f))z & MA16 & 0.38 & 0.86 & 1.08 & 1.26 \\
\hline $\mathrm{BD}+364145$ & $20: 36: 18.21$ & $+37: 25: 02.8$ & $08.5 \mathrm{~V}(\mathrm{n})$ & MA16 & 0.78 & 0.71 & 0.72 & 0.92 \\
\hline ALS 12050 & $21: 55: 15.29$ & $+57: 39: 45.7$ & O5 V((f)) & MA16 & 0.25 & 0.74 & 0.64 & 0.86 \\
\hline
\end{tabular}


Table 1

(Continued)

\begin{tabular}{|c|c|c|c|c|c|c|c|c|}
\hline Object & $\begin{array}{l}\text { R.A. } \\
\text { hh:mm: } \\
\text { ss.ss }\end{array}$ & $\begin{array}{c}\text { decl. } \\
\text { dd:am:as.s }\end{array}$ & Sp. Type & References & $\begin{array}{c}\mathrm{EW}\left(\mathrm{He}_{\mathrm{I}} 4471\right) \\
(\AA)\end{array}$ & $\begin{array}{c}\text { EW(He II 4542) } \\
(\AA)\end{array}$ & $\begin{array}{c}\text { EW(He II 4686) } \\
(\AA)\end{array}$ & $z$ \\
\hline $\mathrm{BD}+552722 \mathrm{~A}$ & $22: 18: 58.63$ & $+56: 07: 23.5$ & $\mathrm{O} 8 \mathrm{Vz}$ & MA16 & 0.73 & 0.72 & 0.84 & 1.14 \\
\hline ALS 12370 & $22: 23: 17.42$ & $+55: 38: 02.3$ & O6.5 Vnn((f)) & MA16 & 0.61 & 0.92 & 0.89 & 0.97 \\
\hline HD $213023 \mathrm{~A}$ & $22: 26: 52.36$ & $+63: 43: 04.9$ & $07.5 \mathrm{Vz}$ & MA16 & 0.69 & 0.72 & 0.86 & 1.20 \\
\hline ALS 12619 & $22: 47: 50.60$ & $+58: 05: 12.4$ & O7 V((f))z & MA16 & 0.71 & 0.81 & 1.10 & 1.36 \\
\hline HD 216532 & $22: 52: 30.56$ & $+62: 26: 25.9$ & $08.5 \mathrm{~V}(\mathrm{n})$ & S11 & 0.93 & 0.69 & 0.77 & 0.83 \\
\hline $\mathrm{BD}+552840$ & $22: 55: 08.49$ & $+56: 22: 58.9$ & $\mathrm{O} 7.5 \mathrm{~V}(\mathrm{n})$ & MA16 & 0.72 & 0.79 & 0.76 & 0.96 \\
\hline HD 217086 & $22: 56: 47.19$ & $+62: 43: 37.6$ & O7 Vnn((f))z & S14 & 0.61 & 0.69 & 0.83 & 1.20 \\
\hline $\mathrm{BD}+602635$ & $23: 53: 05.21$ & $+60: 54: 44.6$ & O6 V((f)) & MA16 & 0.48 & 0.91 & 0.95 & 1.04 \\
\hline
\end{tabular}

Table 2

O Binaries with Single-lined Profiles at the GOSSS Resolution

\begin{tabular}{|c|c|c|c|c|c|c|c|c|}
\hline Object & $\begin{array}{c}\text { R.A. } \\
\text { hh:mm:ss.ss }\end{array}$ & $\begin{array}{c}\text { decl. } \\
\text { dd:am:as.s }\end{array}$ & Sp. Type & References & $\begin{array}{c}\mathrm{EW}\left(\mathrm{He}_{\mathrm{I}} 4471\right) \\
(\AA)\end{array}$ & $\begin{array}{c}\mathrm{EW}\left(\mathrm{He}_{\text {II }} 4542\right) \\
(\AA)\end{array}$ & $\begin{array}{c}\mathrm{EW}\left(\mathrm{He}_{\text {II }} 4686\right) \\
(\AA)\end{array}$ & $z$ \\
\hline V747 Cep & $00: 01: 46.87$ & $+67: 30: 25.1$ & $05.5 \mathrm{~V}(\mathrm{n})((\mathrm{f}))$ & MA16 & 0.39 & 0.70 & 0.66 & 0.94 \\
\hline $\mathrm{HD} 14633 \mathrm{AaAb}$ & $02: 22: 54.29$ & $+41: 28: 47.7$ & ON8.5 V & S11 & 1.01 & 0.78 & 0.72 & 0.71 \\
\hline HD $17520 \mathrm{~A}$ & $02: 51: 14.43$ & $+60: 23: 10.0$ & $08 \mathrm{~V}$ & MA16 & 0.80 & 0.75 & 0.68 & 0.85 \\
\hline HDE 242908 & $05: 22: 29.30$ & $+33: 30: 50.4$ & $\mathrm{O} 4.5 \mathrm{~V}(\mathrm{n})((\mathrm{fc})) \mathrm{z}$ & $\mathrm{S} 14$ & 0.28 & 0.74 & 0.94 & 1.26 \\
\hline HD 46149 & $06: 31: 52.53$ & $+05: 01: 59.2$ & $08.5 \mathrm{~V}$ & S11 & $\ldots$ & $\ldots$ & $\ldots$ & $\ldots$ \\
\hline HD 46573 & $06: 34: 23.57$ & $+02: 32: 02.9$ & $\mathrm{O} 7 \mathrm{~V}((\mathrm{f})) \mathrm{z}$ & S11 & 0.72 & 0.77 & 0.88 & 1.15 \\
\hline 15 Mon $\mathrm{AaAb}$ & $06: 40: 58.66$ & $+09: 53: 44.7$ & O7 V((f))z var & $\mathrm{S} 14$ & 0.70 & 0.67 & 0.78 & 1.12 \\
\hline ALS 85 & $06: 45: 48.84$ & $-07: 18: 46.4$ & $07.5 \mathrm{~V}$ & MA16 & 0.69 & 0.86 & 0.89 & 1.04 \\
\hline HD 53975 & 07:06:35.96 & $-12: 23: 38.2$ & $07.5 \mathrm{Vz}$ & $\mathrm{S} 11$ & 0.75 & 0.70 & 0.83 & 1.11 \\
\hline HD $54662 \mathrm{AB}$ & 07:09:20.25 & $-10: 20: 47.6$ & O7 Vz var? & $\mathrm{S} 14$ & $\ldots$ & $\ldots$ & $\ldots$ & $\ldots$ \\
\hline V467 Vel & $08: 43: 49.81$ & $-46: 07: 08.8$ & $06.5 \mathrm{~V}(\mathrm{n})((\mathrm{f}))$ & MA16 & 0.51 & 0.62 & 0.52 & 0.83 \\
\hline HD 91572 & $10: 33: 12.27$ & $-58: 10: 13.6$ & $06.5 \mathrm{~V}((\mathrm{f})) \mathrm{z}$ & S14 & 0.50 & 0.61 & 0.74 & 1.22 \\
\hline HD 91824 & $10: 34: 46.63$ & $-58: 09: 22.0$ & $\mathrm{O} 7 \mathrm{~V}((\mathrm{f})) \mathrm{z}$ & S14 & 0.60 & 0.78 & 0.89 & 1.14 \\
\hline HD 92206 A & $10: 37: 22.28$ & $-58: 37: 22.8$ & O6 V((f))z & S14 & $\ldots$ & $\ldots$ & $\ldots$ & $\ldots$ \\
\hline HD 93146 A & 10:44:00.16 & $-60: 05: 09.9$ & O7 V((f)) & MA16 & 0.68 & 0.79 & 0.78 & 0.98 \\
\hline HD $93222 \mathrm{AB}$ & $10: 44: 36.25$ & $-60: 05: 28.9$ & O7 V((f)) & MA16 & 0.62 & 0.66 & 0.72 & 1.09 \\
\hline CPD -59 2600 & $10: 44: 41.79$ & $-59: 46: 56.4$ & O6 V((f)) & $\mathrm{S} 14$ & 0.40 & 0.69 & 0.65 & 0.94 \\
\hline $\mathrm{CPD}-592626 \mathrm{AB}$ & $10: 45: 05.79$ & $-59: 45: 19.6$ & $\mathrm{O} 7.5 \mathrm{~V}(\mathrm{n})$ & MA16 & 0.73 & 0.78 & 0.72 & 0.92 \\
\hline HDE $303308 \mathrm{AB}$ & $10: 45: 05.92$ & $-59: 40: 05.9$ & $\mathrm{O} 4.5 \mathrm{~V}((\mathrm{fc}))$ & $\mathrm{S} 14$ & 0.23 & 0.64 & 0.55 & 0.86 \\
\hline CPD -59 2641 & $10: 45: 16.52$ & $-59: 43: 37.0$ & $\mathrm{O} 6 \mathrm{~V}((\mathrm{fc}))$ & S14 & 0.33 & 0.63 & 0.55 & 0.87 \\
\hline HD 101191 & $11: 38: 12.17$ & $-63: 23: 26.8$ & $\mathrm{O} 8 \mathrm{~V}$ & S14 & 0.74 & 0.50 & 0.76 & 1.03 \\
\hline HD $101413 \mathrm{AB}$ & $11: 39: 45.84$ & $-63: 28: 40.1$ & $\mathrm{O} 8 \mathrm{~V}$ & S14 & 0.76 & 0.63 & 0.65 & 0.85 \\
\hline HD 101436 & $11: 39: 49.96$ & $-63: 28: 43.6$ & $06.5 \mathrm{~V}((\mathrm{f}))$ & S14 & $\ldots$ & $\ldots$ & $\ldots$ & $\ldots$ \\
\hline $\mathrm{HD} 123590 \mathrm{AB}$ & $14: 10: 43.97$ & $-62: 28: 44.4$ & $\mathrm{O} 8 \mathrm{~V}$ & S14 & $\ldots$ & $\ldots$ & $\ldots$ & $\ldots$ \\
\hline HD 145217 & $16: 12: 00.30$ & $-50: 18: 20.5$ & $\mathrm{O} 8 \mathrm{~V}$ & MA16 & $\ldots$ & $\ldots$ & $\ldots$ & $\ldots$ \\
\hline HD $150135 \mathrm{AaAb}$ & $16: 41: 19.45$ & $-48: 45: 47.5$ & $06.5 \mathrm{~V}((\mathrm{f})) \mathrm{z}$ & S14 & $\ldots$ & $\ldots$ & $\ldots$ & $\ldots$ \\
\hline HD 152590 & $16: 56: 05.22$ & $-40: 20: 57.6$ & $\mathrm{O} 7.5 \mathrm{Vz}$ & S14 & 0.68 & 0.71 & 0.90 & 1.27 \\
\hline HD $152623 \mathrm{AaAbB}$ & $16: 56: 15.03$ & $-40: 39: 35.8$ & $\mathrm{O} 7 \mathrm{~V}(\mathrm{n})((\mathrm{f}))$ & MA16 & $\ldots$ & $\ldots$ & $\ldots$ & $\ldots$ \\
\hline HD 155913 & $17: 16: 26.34$ & $-42: 40: 04.1$ & $\mathrm{O} 4.5 \mathrm{Vn}((\mathrm{f}))$ & S14 & 0.31 & 0.86 & 0.75 & 0.88 \\
\hline HDE 319699 & $17: 19: 30.42$ & $-35: 42: 36.1$ & $\mathrm{O} 5 \mathrm{~V}((\mathrm{fc}))$ & S14 & 0.25 & 0.73 & 0.57 & 0.79 \\
\hline HDE $319703 \mathrm{BaBb}$ & $17: 19: 45.05$ & $-36: 05: 47.0$ & O6 V((f))z & S14 & 0.47 & 0.60 & 0.71 & 1.19 \\
\hline ALS 19693 & $17: 25: 29.17$ & $-34: 25: 15.7$ & O6 $\operatorname{Vn}((\mathrm{f}))$ & MA16 & 0.38 & 0.76 & 0.74 & 0.97 \\
\hline HD 164536 & $18: 02: 38.62$ & $-24: 15: 19.4$ & $\mathrm{O} 7.5 \mathrm{~V}(\mathrm{n})$ & MA16 & 0.75 & 0.71 & 0.81 & 1.07 \\
\hline 9 Sgr $A B$ & $18: 03: 52.45$ & $-24: 21: 38.6$ & $\mathrm{O} 4 \mathrm{~V}((\mathrm{f}))$ & MA16 & $\ldots$ & $\ldots$ & $\ldots$ & $\ldots$ \\
\hline HD 165246 & 18:06:04.68 & $-24: 11: 43.9$ & O8 V(n) & S14 & 0.81 & 0.59 & 0.75 & 0.93 \\
\hline HD 168075 & $18: 18: 36.04$ & $-13: 47: 36.5$ & $\mathrm{O} 7 \mathrm{~V}((\mathrm{f}))$ & MA16 & $\ldots$ & $\ldots$ & $\ldots$ & $\ldots$ \\
\hline HD $168137 \mathrm{AaAb}$ & $18: 18: 56.19$ & $-13: 48: 31.1$ & $\mathrm{O} 8 \mathrm{Vz}$ & MA16 & 0.72 & 0.72 & 0.88 & 1.22 \\
\hline $\mathrm{BD}-164826$ & $18: 21: 02.23$ & $-16: 01: 00.9$ & $05.5 \mathrm{~V}((\mathrm{f})) \mathrm{z}$ & MA16 & 0.42 & 0.69 & 0.84 & 1.21 \\
\hline V479 Sct & $18: 26: 15.05$ & $-14: 50: 54.3$ & ON6 V((f))z & MA16 & 0.51 & 0.94 & 1.05 & 1.12 \\
\hline Cyg OB2-17 & $20: 32: 50.01$ & $\begin{array}{r}\text { 41:23:44.7 } \\
\end{array}$ & O8 V & MA16 & 0.77 & 0.80 & 0.80 & 1.00 \\
\hline ALS 15115 & $20: 33: 18.05$ & $+41: 21: 36.9$ & $\mathrm{O} 8 \mathrm{~V}$ & MA16 & $\ldots$ & $\ldots$ & $\ldots$ & $\ldots$ \\
\hline Cyg OB2-29 & $20: 34: 13.50$ & $+41: 35: 03.0$ & $07.5 \mathrm{~V}(\mathrm{n})((\mathrm{f})) \mathrm{z}$ & MA16 & 0.62 & 0.62 & 0.79 & 1.27 \\
\hline ALS 15114 & $20: 34: 29.60$ & $+41: 31: 45.4$ & $\mathrm{O} 7.5 \mathrm{~V}(\mathrm{n})(\mathrm{(f}))$ & MA16 & $\ldots$ & $\ldots$ & $\ldots$ & $\ldots$ \\
\hline HD 199579 & $20: 56: 34.78$ & $+44: 55: 29.0$ & $06.5 \mathrm{~V}((\mathrm{f})) \mathrm{z}$ & $\mathrm{S} 11$ & 0.57 & 0.60 & 0.85 & 1.40 \\
\hline $\mathrm{BD}+622078$ & $22: 25: 33.58$ & $+63: 25: 02.6$ & $\mathrm{O} 7 \mathrm{~V}((\mathrm{f})) \mathrm{z}$ & MA16 & 0.62 & 0.82 & 0.92 & 1.13 \\
\hline
\end{tabular}


Table 3

Double-lined Binary Stars in GOSSS

\begin{tabular}{|c|c|c|c|c|c|c|c|c|}
\hline Object & $\begin{array}{c}\text { R.A. } \\
\text { hh:mm:ss.ss }\end{array}$ & $\begin{array}{c}\text { decl. } \\
\text { dd:am:as.s }\end{array}$ & $\begin{array}{c}\text { Sp. Classif. } \\
\text { Prim. + Second. }\end{array}$ & References & $\begin{array}{c}\text { EW } \\
\left(\mathrm{He}_{\mathrm{I}} 4471\right) \\
(\AA) \\
\text { Prim./ } \\
\text { Second. }\end{array}$ & $\begin{array}{c}\text { EW } \\
\text { (He II 4542) } \\
(\AA) \\
\text { Prim./ } \\
\text { Second. }\end{array}$ & $\begin{array}{c}\text { EW } \\
(\mathrm{He} \text { II } 4686) \\
(\AA) \\
\text { Prim./ } \\
\text { Second. }\end{array}$ & $\begin{array}{c}z \\
\text { Prim./ } \\
\text { Second. }\end{array}$ \\
\hline ALS 6967 & 02:12:29.97 & $+59: 54: 04.1$ & $\mathrm{O} 8 \mathrm{~V}+\mathrm{B} 0: \mathrm{V}$ & MA16 & 0.63 & 0.38 & 0.52 & 0.83 \\
\hline $\mathrm{BD}+60497$ & 02:31:57.09 & $+61: 36: 43.9$ & $\begin{array}{c}\mathrm{O} 6.5 \mathrm{~V}((\mathrm{f}))+ \\
\mathrm{O} 8 / \mathrm{B} 0 \mathrm{~V}\end{array}$ & $\mathrm{~S} 11$ & $\ldots$ & $\ldots$ & $\ldots$ & $\ldots$ \\
\hline HD 18326 & $02: 59: 23.17$ & $+60: 33: 59.5$ & $\begin{array}{l}\mathrm{O} 6.5 \mathrm{~V}((\mathrm{f})) \mathrm{z}+ \\
\mathrm{O} 9 / \mathrm{B} 0 \mathrm{~V}:\end{array}$ & S14 & $\cdots$ & $\cdots$ & $\cdots$ & $\cdots$ \\
\hline MY Cam & 03:59:18.29 & $+57: 14: 13.7$ & $\begin{array}{c}05.5 \mathrm{~V}(\mathrm{n})+ \\
\mathrm{O} 6.5 \mathrm{~V}(\mathrm{n})\end{array}$ & MA16 & $0.23 / 0.29$ & $0.78 / 0.36$ & $0.51 / 0.38$ & $0.65 / 1.06$ \\
\hline ALS 8272 & 05:20:00.63 & $+38: 54: 43.5$ & $\begin{array}{c}\mathrm{O} 7 \mathrm{~V}((\mathrm{f}))+\mathrm{B} 0 \\
\text { III-V }\end{array}$ & MA16 & 0.44 & 0.58 & 0.53 & 0.92 \\
\hline HD 48099 & $06: 41: 59.23$ & $+06: 20: 43.5$ & $\begin{array}{c}05.5 \mathrm{~V}((\mathrm{f})) \mathrm{z} \\
+\mathrm{O} 9 \mathrm{~V}\end{array}$ & MA16 & $\cdots$ & $\ldots$ & $\ldots$ & $\ldots$ \\
\hline HD $64315 \mathrm{AB}$ & $07: 52: 20.28$ & $-26: 25: 46.7$ & $\mathrm{O} 5.5 \mathrm{~V}+\mathrm{O} 7 \mathrm{~V}$ & MA16 & $0.34 / 0.37$ & $0.61 / 0.41$ & $0.63 / 0.35$ & $1.03 / 0.84$ \\
\hline HD $92206 \mathrm{C}$ & $10: 37: 18.63$ & $-58: 37: 41.7$ & O8 V(n)z + B0: V & MA16 & 0.46 & 0.45 & 0.53 & 1.17 \\
\hline ALS 15204 & $10: 43: 41.24$ & $-59: 35: 48.2$ & $07.5 \mathrm{Vz}+\mathrm{O} 9: \mathrm{V}$ & MA16 & $\ldots$ & $\ldots$ & $\ldots$ & $\ldots$ \\
\hline HD $93161 \mathrm{~A}^{*}$ & $10: 44: 08.84$ & $-59: 34: 34.5$ & $\mathrm{O} 7.5 \mathrm{~V}+\mathrm{O} 9 \mathrm{~V}$ & S14 & 0.42 & 0.60 & 0.67 & 1.11 \\
\hline HD 93205 & $10: 44: 33.74$ & $-59: 44: 15.5$ & $\mathrm{O} 3.5 \mathrm{~V}(\mathrm{f}))+\mathrm{O} 8 \mathrm{~V}$ & S14 & $\cdots$ & $\cdots$ & $\cdots$ & $\cdots$ \\
\hline CPD -59 2591 & $10: 44: 36.69$ & $-59: 47: 29.6$ & O8 Vz + B0.5: V: & MA16 & 0.58 & 0.58 & 0.69 & 1.19 \\
\hline V572 Car & $10: 44: 47.31$ & $-59: 43: 53.2$ & $\begin{array}{l}\mathrm{O} 7.5 \mathrm{~V}(\mathrm{n})+ \\
\mathrm{B} 0 \mathrm{~V}(\mathrm{n})\end{array}$ & MA16 & 0.63 & 0.44 & 0.57 & 0.91 \\
\hline HD 93343 & $10: 45: 12.22$ & $-59: 45: 00.4$ & $\mathrm{O} 8 \mathrm{~V}+\mathrm{sec}$ & MA16 & $\ldots$ & $\ldots$ & $\ldots$ & $\ldots$ \\
\hline CPD -59 2635 & $10: 45: 12.72$ & $-59: 44: 46.2$ & $\mathrm{O} 8 \mathrm{~V}(\mathrm{n})+09.5 \mathrm{~V}$ & S14 & 0.57 & 0.55 & 0.42 & 0.72 \\
\hline $\mathrm{CPD}-592636 \mathrm{AB}$ & $10: 45: 12.87$ & $-59: 44: 19.2$ & $\mathrm{O} 8 \mathrm{~V}+\mathrm{O} 8 \mathrm{~V}$ & S14 & $\ldots$ & $\ldots$ & $\ldots$ & $\cdots$ \\
\hline V662 Car & $10: 45: 36.32$ & $-59: 48: 23.2$ & O5 V(n)z + B0: V & MA16 & 0.24 & 0.77 & 0.85 & 1.11 \\
\hline HDE 305525 & 10:46:05.70 & $-59: 50: 49.4$ & $\begin{array}{c}\mathrm{O} 5.5 \mathrm{~V}(\mathrm{n})((\mathrm{f})) \mathrm{z} \\
+\mathrm{sec}\end{array}$ & S14 & $\cdots$ & $\cdots$ & $\cdots$ & $\cdots$ \\
\hline ALS $18551^{*}$ & $10: 58: 17.68$ & $-61: 12: 03.5$ & $\begin{array}{c}\mathrm{O} 4.5 \mathrm{~V}(\mathrm{n}) \mathrm{z}+ \\
\mathrm{O} 4.5 \mathrm{~V}(\mathrm{n}) \mathrm{z}\end{array}$ & MA16 & $0.11 / 0.07$ & $0.47 / 0.45$ & $0.44 / 0.38$ & $0.93 / 0.85$ \\
\hline $\begin{array}{l}\text { 2MASS J10583238- } \\
\quad 6110565\end{array}$ & $10: 58: 32.39$ & $-61: 10: 56.5$ & $\begin{array}{l}\mathrm{O} 5 \mathrm{~V}((\mathrm{f}))+ \\
\mathrm{O} 7 \mathrm{~V}((\mathrm{f}))\end{array}$ & MA16 & $\cdots$ & $\cdots$ & $\cdots$ & $\cdots$ \\
\hline HD 97484 & 11:12:04.50 & $-61: 05: 42.9$ & $\begin{array}{l}07.5 \mathrm{~V}((\mathrm{f}))+ \\
07.5 \mathrm{~V}((\mathrm{f}))\end{array}$ & MA16 & $0.37 / 0.34$ & $0.46 / 0.33$ & $0.34 / 0.27$ & $0.74 / 0.79$ \\
\hline HD 100213 & $11: 31: 10.93$ & $-65: 44: 32.1$ & $\mathrm{O} 8 \mathrm{~V}(\mathrm{n})+\mathrm{B} 0 \mathrm{~V}(\mathrm{n})$ & MA16 & 0.54 & 0.45 & 0.53 & 0.98 \\
\hline HD $101131 \mathrm{AB}$ & $11: 37: 48.44$ & $-63: 19: 23.5$ & $\begin{array}{l}\text { O5.5 V((f)) } \\
+ \text { O8: V }\end{array}$ & S14 & $\cdots$ & $\cdots$ & $\cdots$ & $\cdots$ \\
\hline Тус 7370-00460-1 & $17: 18: 15.40$ & $-34: 00: 05.9$ & O6 V V(ff)) + O8 V & MA16 & $0.33 / 0.22$ & $0.41 / 0.37$ & $0.43 / 0.38$ & $1.04 / 1.03$ \\
\hline HDE $319703 \mathrm{~A}$ & $17: 19: 46.16$ & $-36: 05: 52.4$ & $\mathrm{O} 7 \mathrm{~V}((\mathrm{f}))+09.5 \mathrm{~V}$ & MA16 & $\cdots$ & $\cdots$ & $\ldots$ & $\ldots$ \\
\hline HD 159176 & $17: 34: 42.49$ & $-32: 34: 54.0$ & $\begin{array}{l}\mathrm{O} 7 \mathrm{~V}((\mathrm{f}))+ \\
\mathrm{O} 7 \mathrm{~V}((\mathrm{f}))\end{array}$ & S14 & $0.34 / 0.42$ & $0.39 / 0.38$ & $0.36 / 0.32$ & $0.94 / 0.75$ \\
\hline HD 161853 & $17: 49: 16.56$ & $-31: 15: 18.1$ & O8 V(n)z + B & S14 & 0.66 & 0.52 & 0.80 & 1.20 \\
\hline Herschel 36 & $18: 03: 40.33$ & $-24: 22: 42.7$ & O7: V + sec & S14 & $\ldots$ & $\cdots$ & $\ldots$ & $\cdots$ \\
\hline HD 165052 & $18: 05: 10.55$ & $-24: 23: 54.8$ & $\mathrm{O} 6 \mathrm{Vz}+\mathrm{O} 8 \mathrm{Vz}$ & MA16 & $\ldots$ & $\cdots$ & $\cdots$ & $\cdots$ \\
\hline HD 165921 & 18:09:17.70 & $-23: 59: 18.3$ & O7 V(n)z + B0: V: & S14 & 0.42 & 0.69 & 0.72 & 1.04 \\
\hline ALS 4923 & $18: 19: 28.43$ & $-15: 18: 46.2$ & $08.5 \mathrm{~V}+08.5 \mathrm{~V}$ & MA16 & $0.36 / 0.33$ & $0.32 / 0.32$ & $0.39 / 0.28$ & $1.09 / 0.84$ \\
\hline HD 175514 & $18: 55: 23.12$ & $+09: 20: 48.1$ & $\mathrm{O} 7 \mathrm{~V}(\mathrm{n})((\mathrm{f})) \mathrm{z}+\mathrm{B}$ & MA16 & $\cdots$ & $\cdots$ & $\cdots$ & $\cdots$ \\
\hline HD 194649 AB & $20: 25: 22.12$ & $+40: 13: 01.1$ & $06.5 \mathrm{~V}((\mathrm{f}))+\mathrm{sec}$ & MA16 & $\cdots$ & $\cdots$ & $\ldots$ & $\cdots$ \\
\hline Cyg OB2-73 & $20: 34: 21.93$ & $+41: 17: 01.6$ & $\mathrm{O} 8 \mathrm{Vz}+\mathrm{O} 8 \mathrm{Vz}$ & MA16 & $0.39 / 0.28$ & $0.27 / 0.27$ & $0.49 / 0.32$ & $1.26 / 1.14$ \\
\hline HD $206267 \mathrm{AaAb}$ & $21: 38: 57.62$ & $+57: 29: 20.6$ & $\begin{array}{c}\text { O6.5 V((f)) }+ \\
\text { O9/B0 V }\end{array}$ & S11a & 0.52 & 0.75 & 0.53 & 0.71 \\
\hline $\mathrm{BD}+552722 \mathrm{C}$ & $22: 18: 59.88$ & $+56: 07: 18.9$ & O7 V(n)z + B & MA16 & 0.51 & 0.72 & 0.80 & 1.11 \\
\hline HD 215835 & $22: 46: 54.11$ & $+58: 05: 03.5$ & $\begin{array}{l}05.5 \mathrm{~V}((\mathrm{f}))+ \\
\mathrm{O} 6 \mathrm{~V}(\mathrm{ff}))\end{array}$ & S14 & $0.13 / 0.37$ & $0.50 / 0.40$ & $0.41 / 0.38$ & $0.81 / 0.96$ \\
\hline ALS 12688 & $22: 55: 44.94$ & $+56: 28: 36.7$ & $05.5 \mathrm{~V}(\mathrm{n})((\mathrm{fc}))+\mathrm{B}$ & MA16 & 0.35 & 0.64 & 0.50 & 0.79 \\
\hline
\end{tabular}

temperature is $37 \mathrm{kK}$ (which may reasonably be associated with an $\mathrm{O} 7$ spectral type) can be morphologically classified either as $\mathrm{Vz}$ or "normal" class $\mathrm{V}$, depending on the value of $v \sin i$. Again, in this "low temperature" regime $\left(T_{\text {eff }} \leqslant 37 \mathrm{kK}\right)$, rapid rotation increases the relevant $\mathrm{CD}$ ratio, thus favoring the $C D$ based $\mathrm{Vz}$ spectral classification.
On the other hand, the relation between the central intensities and the equivalent widths of the spectral lines depends on various parameters, which leads to the following "paradoxical" result. Let us consider one of the principal horizontal (i.e., spectral-class or temperature) classification criteria for $\mathrm{O}$ stars, i.e., the ratio between the intensities of the $\mathrm{He} \mathrm{I} \lambda 4471$ and 
He $\mathrm{II} \lambda 4542$ lines. By definition, $\mathrm{He}_{\mathrm{I}} \lambda 4471$ is equal in strength to $\mathrm{He}$ II $\lambda 4542$ for type $\mathrm{O} 7$.

Using FASTWIND model predictions, we analyzed the variation of the ratios between the CDs and the EWs of the former lines as a function of effective temperature (see Figure 3). We found that for the considered values of the projected rotational velocity, surface gravity, resolution, and wind strength, the CDs of the two lines are equal at $T_{\text {eff }}=T_{1} \approx 39 \mathrm{kK}$, whereas the equality between their EWs occurs at the much lower temperature of $T_{\mathrm{eff}}=T_{2} \approx 36.5 \mathrm{kK}$. Current calibrations (Martins et al. 2005; Simón-Díaz et al. 2014), assign those temperatures to spectral types that differ by at least one subtype. It then seems essential to define a consistent classification criterion.

Walborn \& Fitzpatrick (1990) stated that the eye is more sensitive to the EWs of the absorption lines in the photographic spectrograms, but to the CDs in the digital data. Thus, systematic and random differences between the results of the two techniques may be expected. Since EWs cannot be estimated visually in digital data, measurements are indicated as the preferred approach.

\subsection{A New "Quantitative” Classification Criterion}

Considering the above complications in using only eyeestimated $\mathrm{CD}$ ratios to define the $\mathrm{Vz}$ characteristic, the necessity of taking into account an additional quantitative criterion, for example, based on the EWs of the relevant lines, appears to be very reasonable. ${ }^{14} \mathrm{~A}$ first attempt of quantification was done by $\mathrm{S} 14$, where the $\mathrm{Vz}$ phenomenon was defined by a ratio

$$
z=\frac{\operatorname{EW}(\text { He II } \lambda 4686)}{\operatorname{Max}[\operatorname{EW}(\text { He I } \lambda 4471), \operatorname{EW}(\text { He II } \lambda 4542)]}
$$

greater than $\sim 1.0$. This recalibration was obtained by measuring the EWs of the former lines in the main-sequence standard stars. In order to have a deeper insight into the behavior of the $z$ characteristic, we have measured the EWs and CDs of the three relevant spectral lines for a large sample of objects selected from the class V stars in the GOSSS database (see Sections 3 and 4.3 for details about the sample selection).

Because the classification categories are discrete, whereas the phenomena are continuous, spectral classification sometimes requires interpolations or compromises. The Vz classification is not an exception, and the situation may be critical for these stars. Marginal $\mathrm{O} \mathrm{Vz}$ classifications may be generating confusion in the general picture, and might be, at least in part, the source of the recent controversy regarding the relation between the $z$ characteristic and the youth of the objects. Therefore, in this work, we "strengthen" the definition of the $\mathrm{Vz}$ phenomenon by increasing the value of the $z$ parameter defined by Equation (1) to 1.10, just to avoid those borderline, often unclear cases. An $\mathrm{O}$ dwarf will then be classified as $\mathrm{Vz}$

\footnotetext{
${ }^{14}$ We should mention here that in parallel with the morphological method, an alternative spectral classification method based on the ratios of the EWs of certain lines was developed by Conti and collaborators, and was subsequently refined by Mathys (e.g., Conti \& Alschuler 1971; Conti \& Leep 1974; Mathys 1988, 1989). However, this quantitative system is calibrated against the standard MK spectral types and does not include quantitative criteria for exceptional objects like the $\mathrm{O}$ Vz stars, which have been defined exclusively on a morphological basis.
}

when

$$
z=z_{4542}=\frac{\mathrm{EW}(\text { He II 4686) }}{\mathrm{EW}(\text { He II 4542) }} \geqslant 1.10
$$

at spectral types earlier than or equal to O6.5, and

$$
z=z_{4471}=\frac{\mathrm{EW}(\mathrm{He} \text { II 4686) }}{\mathrm{EW}\left(\mathrm{He} \mathrm{I}_{\mathrm{I}} 471\right)} \geqslant 1.10
$$

at types later than or equal to O7.5. At the boundary type $\mathrm{O} 7$, $z_{4542}$ and $z_{4471}$ must both be greater than or equal to 1.10 .

The new critical value for the $z$ parameter was adopted by MA16 in the third installment of the GOSSS project. Besides the reclassification of the dwarfs from S11 and S14 (several of which have changed their " $z$ status" due to the new more strict requirement of $z \geqslant 1.10$ ), this change implied updates to the grid of standard stars used for this task. Moreover, it led to the definition, for the first time, of $\mathrm{O}$ Vz standard spectra (see Table 2 in MA16). Within the MK scheme of spectral classification, having a complete grid of accurately selected standards is the key for reliable classifications. The quantitative condition $z \geqslant$ 1.10 may also be used to confirm whether or not the $z$ qualifier must be assigned, allowing unclear marginal cases to be objectively resolved.

Spectral classifications and EW measurements for the presumably single stars, single-lined binaries, and double-lined binaries included in our sample are compiled in Tables 1-3, respectively. For the three tables, column 4 contains the new GOSSS spectral classifications taking into account the above criteria (reference quoted in column 5). Columns 6,7 , and 8 show the EW measurements for the three He lines involved in the $\mathrm{Vz}$ phenomenon, and column 9 includes the $z$ parameter computed from Equation (1).

The spectral classifications in the three main GOSSS papers (S11, S14, and MA16) are obtained by a combination of the classical morphological method of visual inspection of the spectra, predominantly determined by the CDs of the lines in the digital data, and the use of MGB (Maíz Apellániz et al. 2012, 2015), which is an interactive software designed specifically for the project. MGB compares the observed spectrum with a grid of low $v \sin i$ standards that can be rotationally broadened to simulate the $(n), n, n n$, and nnn classification suffixes. Therefore, MGB goes beyond the visual $\mathrm{CD}$ and line-width estimates, producing results based on the complete profiles of the lines, and compensating for the dependence of the CDs on rotation. The results of the two techniques have been compared and the final classifications adjusted slightly in some cases. In a future project, these classifications will be compared with the measured EW ratios. We have also verified that there is overall good agreement between the MGB results and the EW determinations of the $z$ characteristic once an appropriate grid of standards is applied. Only for two cases, the double-lined binaries indicated with an asterisk in Table 3, are the GOSSS classifications not in accord with the measured $z$ parameter.

It is interesting to note that in the context of the present quantification of the $z$ characteristic, we became aware of several spectra classified as type $\mathrm{V}$ with excessively small values of the $z$ parameter, i.e., in the range $0.5-0.7$. A number of them were of types O4-O5.5, and inspection from this point of view revealed Of morphologies in the GOSSS data which are well intermediate between most class $\mathrm{V}$ spectra and the class III standards, in terms of both the He II $\lambda 4686$ absorption 

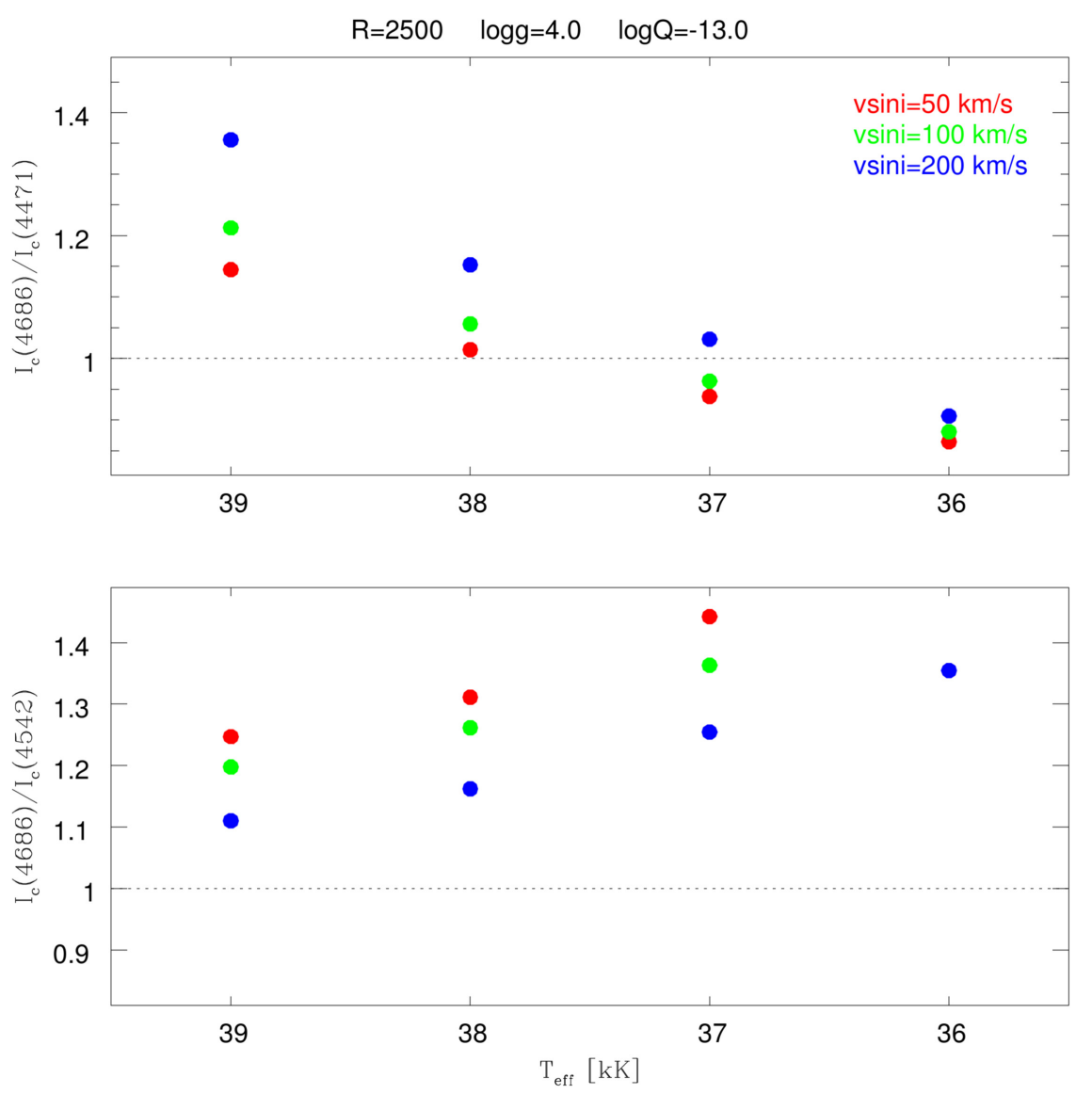

Figure 2. Effect of stellar rotation on the Vz phenomenon defined from the relative depths of the He lines, as shown from FASTwinD model predictions. The intensity $I_{c}$ is defined as $I_{c}=1-F_{c}$, where $F_{c}$ is the flux in the core and indicates the central depth of the line. A star with effective temperature $T_{\text {eff }}=37 \mathrm{kK}$ (corresponding approximately to spectral type $\mathrm{O} 7$ ) may be classified either as a $\mathrm{Vz}$ or normal dwarf depending on its projected rotational velocity.

and $\mathrm{N}_{\text {IIII }} \lambda 4640$ emission strengths. This finding led to the extension of the range of spectral types for which luminosity class IV is defined. The reader is referred to paper MA16 for details about this recent change.

\subsection{Measurements of the Spectral Lines}

As mentioned before, we measured the EWs of the three spectral lines involved in the $\mathrm{Vz}$ phenomenon for a large sample of dwarfs selected from the GOSSS database. In Section 3, we explained that according to the goals of the present work, we excluded stars with spectral types later than O8.5 and divided the 223 remaining dwarfs in the GOSSS database into five different categories. With single-lined features and no evidence of binarity, the 132 objects included in category 1 represent the "simplest" case; all of them have been measured and considered in the analysis related to the $\mathrm{Vz}$ class. Their measurements are presented in Table 1 . The 23 double-lined binaries in category 3 are also included in the analysis. The measurements of their spectra required the use of deblending methods. As only stars with spectral types earlier than $\mathrm{O} 9$ are relevant to the present study, only a few secondary components, specifically nine objects, have been measured (see for example MY Cam = BD+56 864 in Table 3).

On the other hand, as they are unmeasurable, none of the 15 double-lined binaries in group 4 could be considered for the statistics. The peculiar objects in group 5 have not been included either in order to avoid possible sources of noise. This category comprises a total of 8 objects. Finally, group 2 represents a middle situation, as 33 of its 45 binaries have been measured and included in the analysis, whereas 12 have not (see Table 2). We recall that this category comprises singlelined (at GOSSS moderate resolution) spectra of known binary systems. The criterion used to discriminate the useful cases from those that should not be considered for the statistics is the following: the contribution of the secondary to the composite spectrum must be sufficiently small that the measurements obtained from the single spectral features are representative of the primary star. In other words, the presence of the secondary does not alter the resulting spectral $z$ or non- $z$ classification.

To measure the EWs in our spectra, we first attempted to fit (semi-automatically) a Voigt function to the line profiles. This method lead to satisfactory fits for the $\mathrm{He}$ I $\lambda 4471$ and He II $\lambda 4686$ lines, but not for the He II $\lambda 4542$ line, so that the latter necessarily had to be measured manually, i.e., by numerical integration of the line profile between two points selected "by hand" on the continuum. For the sake of uniformity in methodology, we also manually measured the other two He features. It must be recognized that the procedure of rectifying a spectrum is not a trivial task, especially at low resolution, and that local rectification errors, even small ones, can significantly affect the intensities of the spectral features. These uncertainties will impact all of the methods of spectral classification, with no exception for those based on EW measurements. In particular, the rectification of many $\mathrm{O}$ spectra around the He II 4542 line is rather complex, as this feature is 

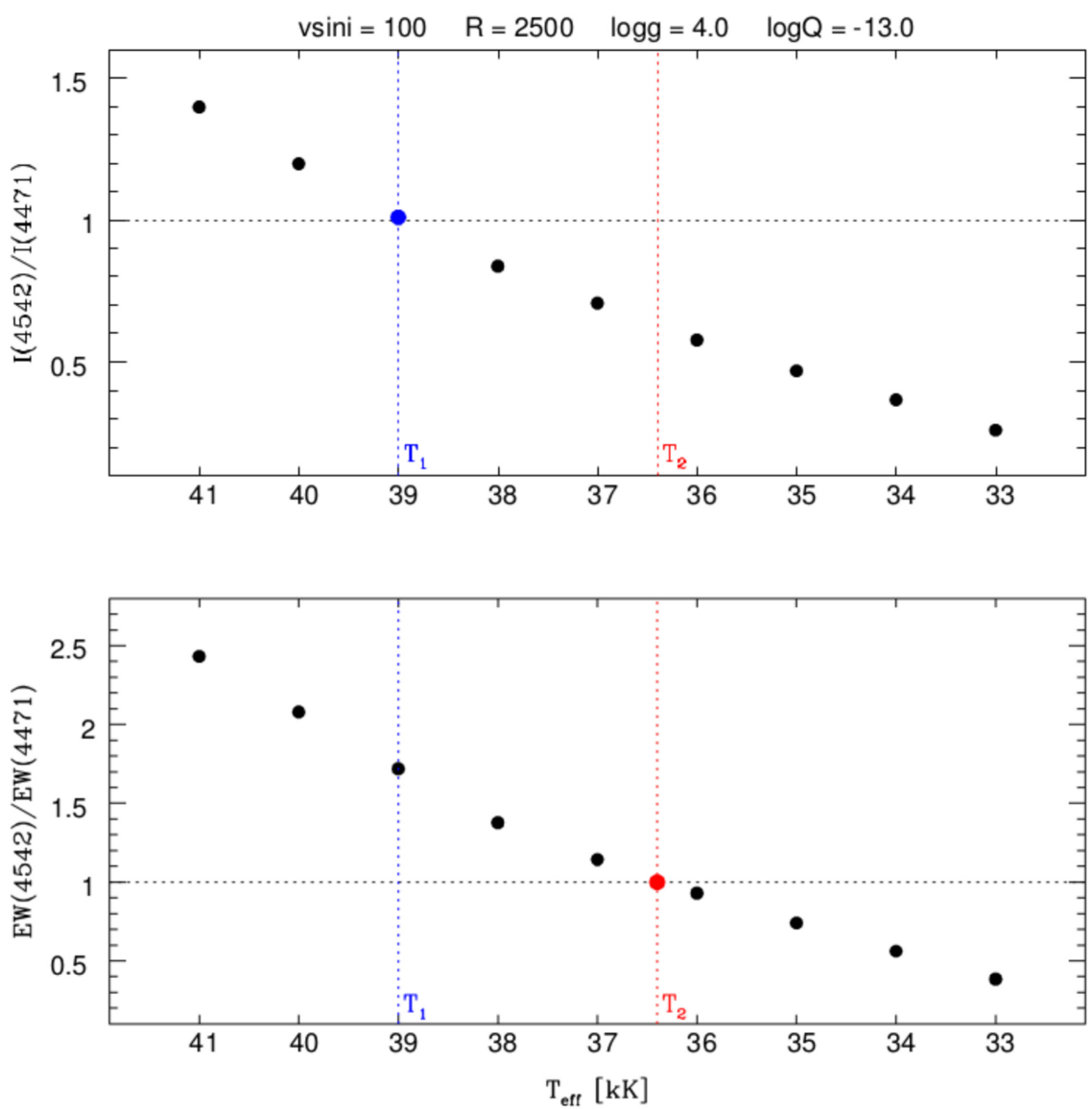

Figure 3. Top panel: variation of the ratio between the CDs of the He I $\lambda 4471$ and $\mathrm{He}$ II $\lambda 4542$ lines, as a function of $T_{\text {eff }}$, as shown by the FASTwIND model predictions. Bottom panel: the same for the ratio of EWs. Fixed values of the projected rotational velocity, surface gravity, resolution, and wind strength have been considered. $T_{1}$ and $T_{2}$ indicate the effective temperature suitable for the $\mathrm{O} 7$ spectral type when the latter is defined from the CDs or EWs ratios, respectively.

intrinsically broader than other $\mathrm{He}$ lines, also showing relatively extended wings. Additionally, in most spectra, the blue wing appears blended with a set of $\mathrm{N}$ III features (4531-4535-4540 ̊). Its measurement was thus the most sensitive. In many cases, we integrated the red half of the profile and considered the double of that value as the best estimate for the total EW. This effect is particularly important for the extreme cases of N-enhanced stars, such as HD 12993 and HD 110360 , illustrated in Figure 4. Note that disregarding this blend would enlarge the $\operatorname{EW}\left(\mathrm{He}_{\mathrm{II}} \lambda 4542\right)$, with a consequent decrease of the $z$ parameter (Equation (1)), thus disfavoring the $z$ characteristic.

Because of the reasons explained in the previous paragraph, the errors in the EW measurements cannot be the same for all of the studied lines. For the usually well-behaved profiles of the He I $\lambda 4471$ and $\mathrm{He}$ II $\lambda 4686$ features, the mean absolute error of the EW can be estimated as $\sigma_{4471,4686} \leqslant 0.02 \AA$. Instead, for the more problematic He II $\lambda 4542$ line, absolute errors can be somewhat larger. Typical values are around $0.03 \AA$ or smaller but, to be conservative, we adopt a mean value of $\sigma_{4542}<0.05 \AA$. The most critical cases regard the EWs of the individual components of double-lined binaries, as deblending methods of measurement generally involve larger errors. For those objects, absolute error values can reach $0.1 \AA$. For relative errors of $2 \%$ to $7 \%$ in the EW measurements, the relative error in the $z$ parameter can be estimated in the range $5 \%-10 \%$.

\subsection{The Effect of Binaries}

The high degree of multiplicity is a striking characteristic of massive stars (Mason et al. 2009; Barbá et al. 2010; Sana et al. 2013, 2014; Sota et al. 2014), and also one of the most troublesome points when studying these objects. Unknown binaries can easily give rise to wrong conclusions. With respect to the Vz classification, the most critical effect is that they may produce false $\mathrm{O} \mathrm{Vz}$ spectra. We have identified a few stars from the GOSSS database that appear to be nice intermediate $\mathrm{O}$ Vz but have subsequently been resolved into a pair composed of an early and late component by means of either other GOSSS spectra or additional high-resolution observations. In such an $\mathrm{O}$ binary, the early component dominates $\mathrm{He}$ II $\lambda 4542$ and the late component dominates $\mathrm{He}_{\mathrm{I}} \lambda 4471$, but both contribute comparably to the enhanced $\mathrm{He}$ II $\lambda 4686$. The case of the multiple system HD 64315 illustrates this situation (see Figure 5). This star is composed of at least two (probably more) components (Mason et al. 2009; Lorenzo et al. 2010; Tokovinin et al. 2010) but, if its spectral lines were measured during the orbital conjunction, then the obtained $z$ parameter would reach 1.14 , and hence the star would be classified as Vz. Given the high binary frequency among $\mathrm{O}$ stars, one may expect not a few $\mathrm{HD}$ 64315-like cases in which a hidden multiplicity is the actual origin for the observed $\mathrm{Vz}$ spectrum.

We have been extremely careful when probing the binary nature of our sample objects. Within the GOSSS database, spectra obtained in more than one epoch are available for many 


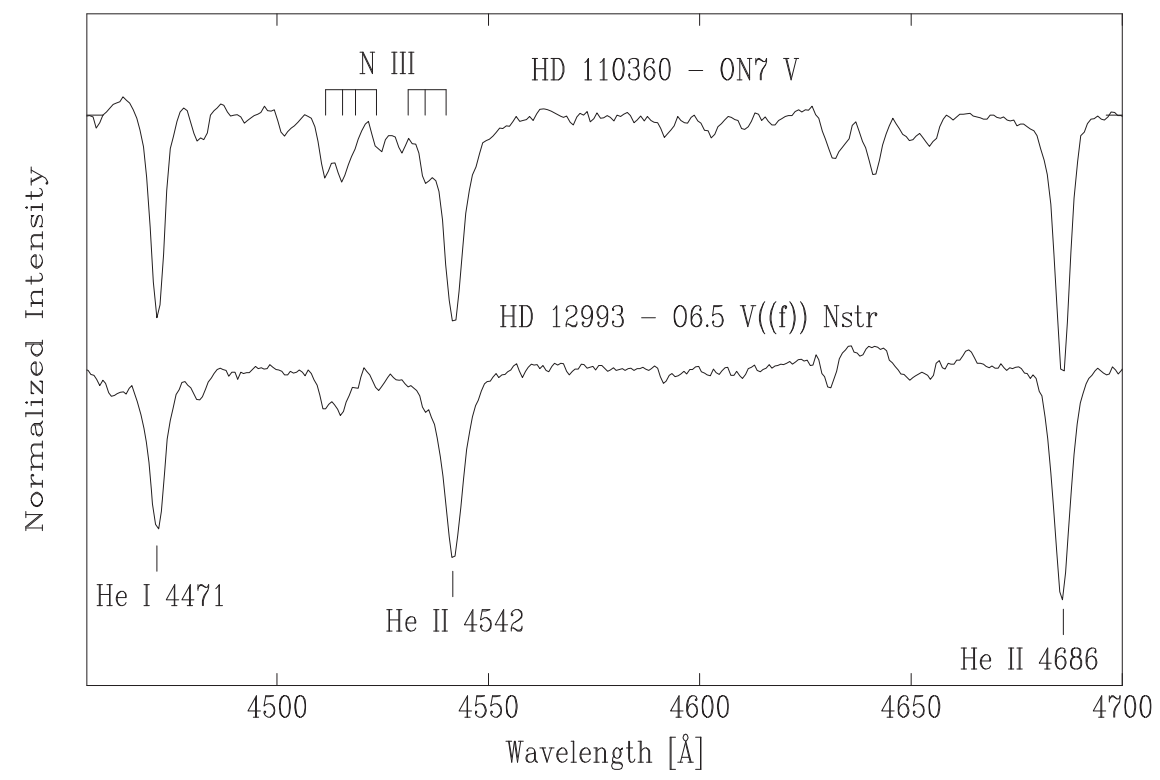

Figure 4. Examples of $\mathrm{He}$ пा $\lambda 4542$ profiles whose EW measurement requires special care, due to the blend with unusually strong $\mathrm{N}$ III lines at $4531-4535-4540 \AA$.

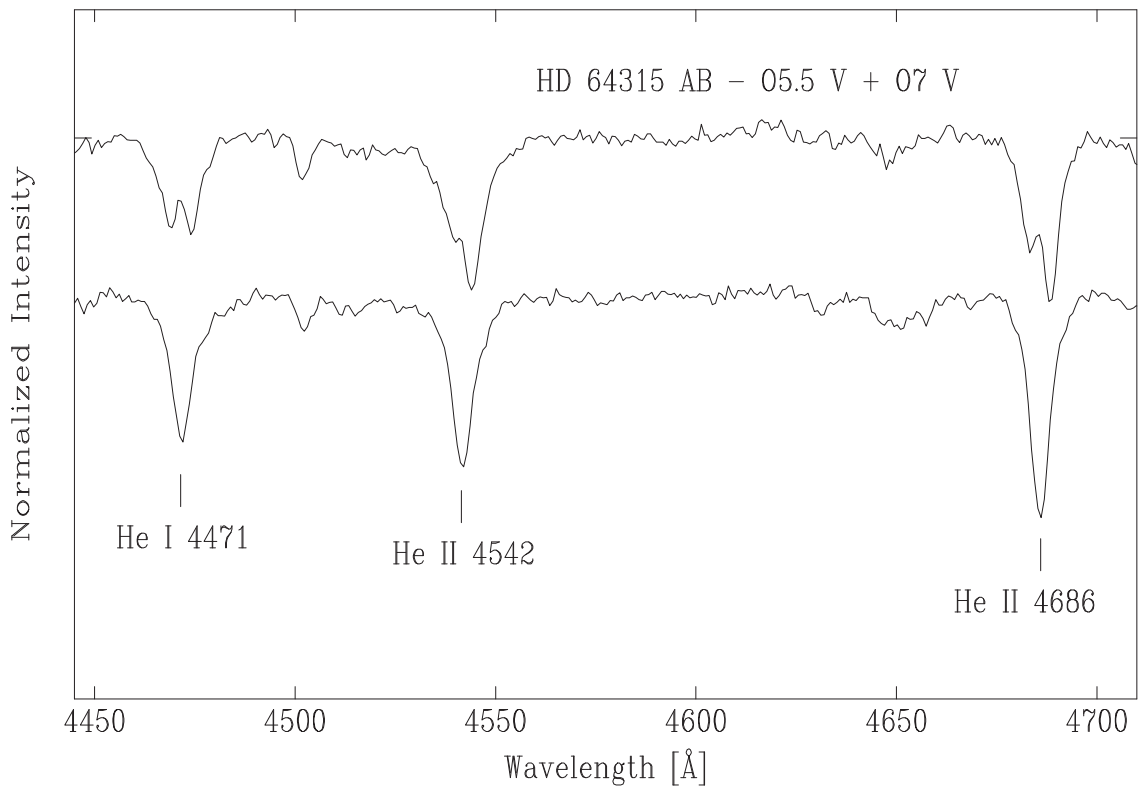

Figure 5. Two spectra of the multiple system HD 64315 illustrating how binaries may produce false $\mathrm{O}$ Vz spectra. If measured during the orbital conjunction, the $z$ parameter of the composite spectrum is $z=1.14$.

stars, which allowed the identification of some spectroscopic binaries. However, we still expected a likely important fraction of unresolved binaries among our sample due to the limited resolution of the data. Therefore, in order to identify as many binaries as possible, we carefully searched for information from the literature, and also took into account results from highresolution surveys coordinated with GOSSS like OWN and IACOB. In fact, these high-resolution monitoring programs appear to be an essential complement for a correct interpretation of the observed statistics.

The misleading origin for some $\mathrm{O} V z$ spectra provided by unknown binaries may obviously be confusing the statistical interpretations. Even if they do not produce false $\mathrm{Vz}$ objects, unresolved binaries may be "blurring" the statistical numbers by leading us to count one object when actually there are two. Among our sample of $223 \mathrm{O}$ dwarfs, we have identified 45 objects whose spectra are single lined at GOSSS resolution (group 2), although they are known to be binaries from higherresolution spectroscopic or imaging data. A recent result by Sana et al. (2014) claims a fraction of $100 \%$ for the luminosity class $\mathrm{V}$ stars that have a bound companion within 30 mas. So the key question is how many of the 132 stars that are assumed to be single in this work are actually single. Further investigation is needed, but meanwhile we must deal with this uncertainty.

\section{THE GOSSS O V AND O VZ POPULATIONS}

Having addressed the methods applied and the problems arising in their application, we now analyze the resulting populations of $\mathrm{OV}$ and $\mathrm{O} \mathrm{Vz}$ stars in the GOSC. 


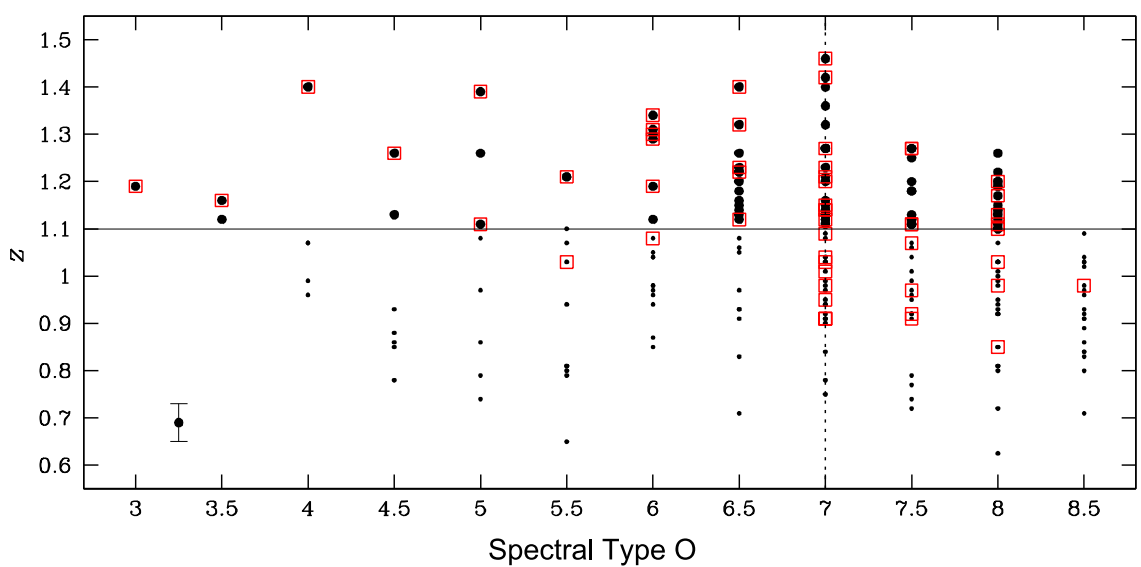

Figure 6. The $z$ parameter computed from the EWs measured in the GOSSS class V spectra as a function of spectral type. The red squares surround O Vz classified previously to the introduction of the quantitative criterion proposed in this paper. The solid line at $z=1.10$ indicates the current threshold to assign the $z$ qualifier. Only the points above this line correspond to the newly classified $\mathrm{O}$ Vz. The representative point with error bars on the lower left corner of the figure illustrates the typical uncertainty in the $z$ parameter.

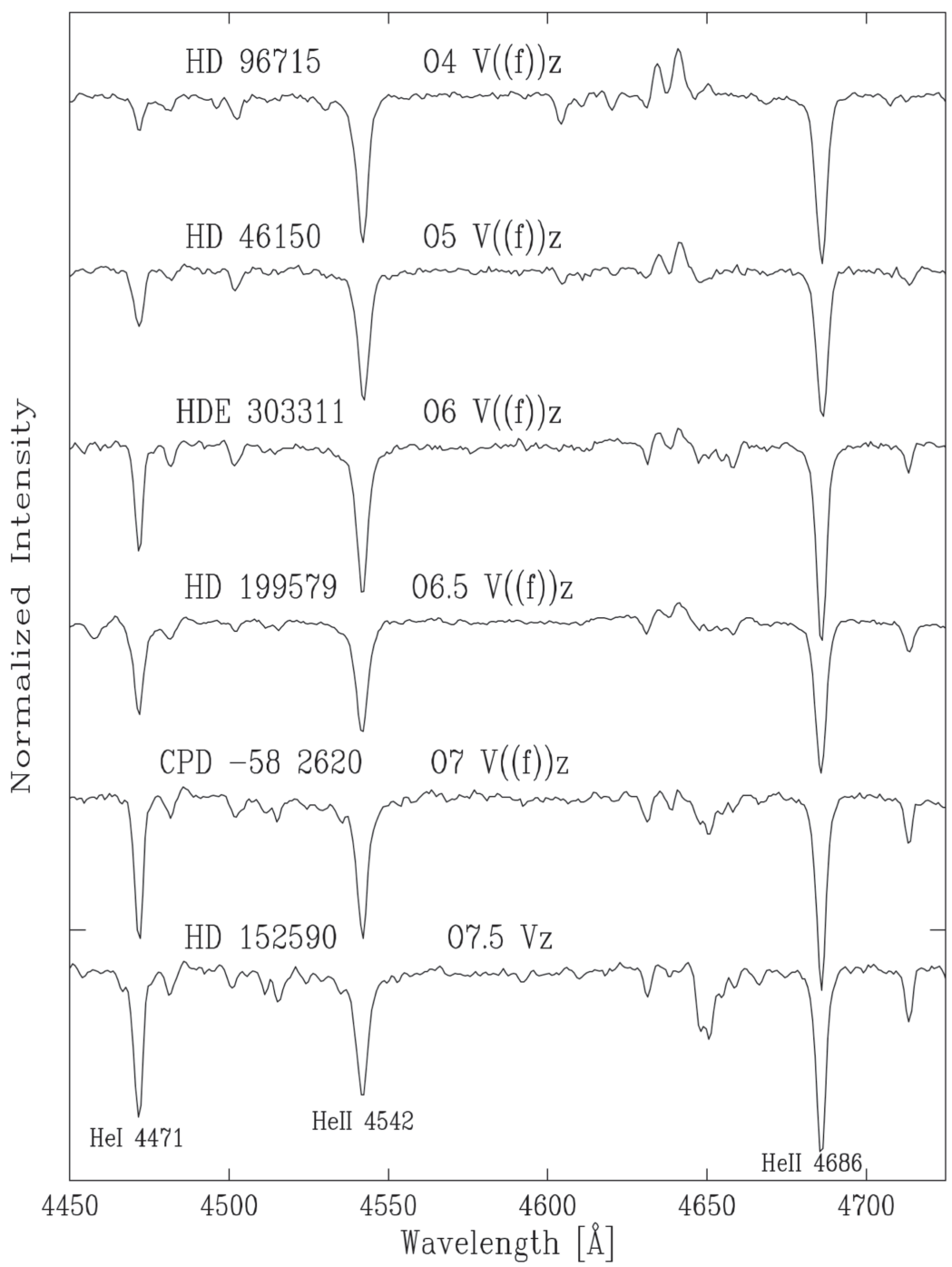

Z

Figure 7. Examples of GOSSS spectra for some of the most extreme cases belonging to the $\mathrm{O}$ Vz class. The rectified spectrograms are separated by 0.2 continuum units. The corresponding $z$ parameter is indicated to the right of each spectrum.

We recall that our $223 \mathrm{O}$ dwarfs were divided into different categories according to their binary status and the appearance of their spectra, and that not all of them could be measured and included in the present analysis (see Sections 3 and 4.3). After "cleaning" the sample, our final group of study, i.e., those stars for which a confident value of the $z$ parameter can be derived, 

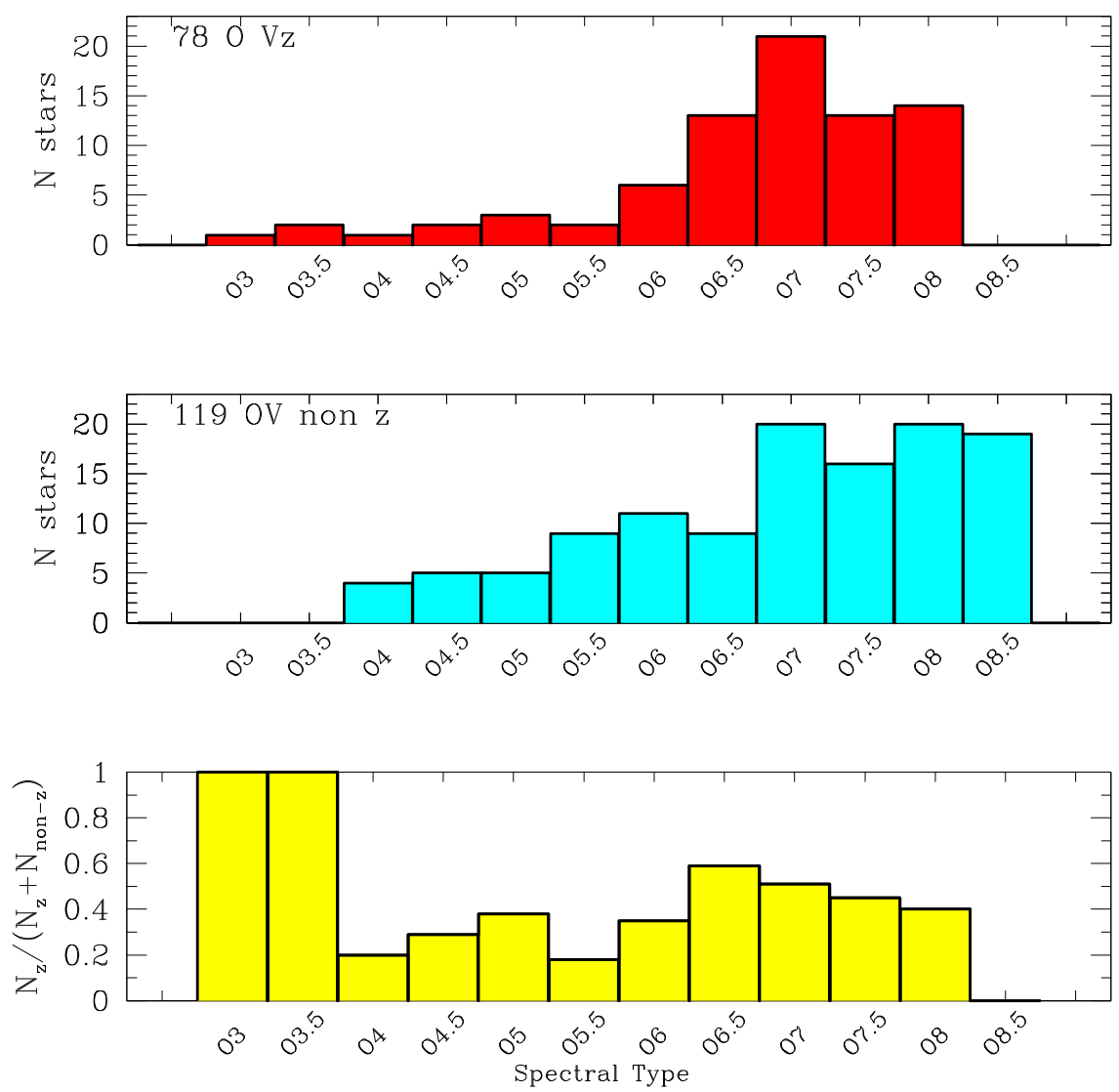

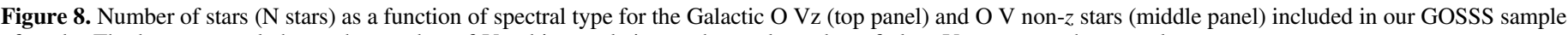
of study. The bottom panel shows the number of $\mathrm{Vz}$ objects relative to the total number of class $\mathrm{V}$ stars at each spectral type.

decreased to 132 presumably single stars, plus 56 binaries or higher-order multiple systems, 23 of which are double-lined at the moderate GOSSS resolution, allowing for individual measurements of nine additional secondary components. A total of 197 individual stars with spectral types earlier than O8.5 were measured, allowing us to apply both the classical morphological and the new quantitative criteria for their spectral classification. We restrict our sample to this set for the statistical considerations on the $\mathrm{O} \mathrm{V}$ and $\mathrm{O} \mathrm{Vz}$ populations.

In Figure 6, the $z$ parameter computed from the EWs of the 197 measured stars (along with additional information described afterwards) is plotted as a function of spectral type. Note the large range of $z$ values observed for most of the spectral types. The $\mathrm{O}$ dwarfs originally classified as $\mathrm{Vz}$ by $\mathrm{S} 11$ and S14 have been marked with red squares. We recall that the former spectral classifications were obtained regardless of the quantitative criterion proposed in the present work. In the same figure, we have marked with a solid line the condition $z \geqslant 1.10$, which represents the threshold to assign the $z$ qualifier proposed here. Only those $\mathrm{O}$ dwarfs whose $z$ parameters fall above this line are now classified as Vz. Slightly larger black circles denote these cases. The representative point on the lower left corner of the figure shows the typical uncertainty in the $z$ parameter. Note that for objects with $z$ close to the threshold, measurement errors might change their " $z$ status." Some examples of spectrograms, particularly for the most extreme cases, are shown in Figure 7.

The spectral type distribution observed for the newly classified $\mathrm{O} \mathrm{Vz}$ stars is presented in the top panel of Figure 8. For comparison, an analogous distribution for the "normal" class $\mathrm{V}$ objects spanning the same spectral type range is included in the middle panel of the same figure. The bottom panel shows relative differences between the two populations for each spectral type.

With the addition of the new quantitative classification criterion, the $\mathrm{O} \mathrm{Vz}$ population decreased to 78 members, and thus they represent $\sim 40 \%$ of the class $\mathrm{V}$ stars in the relevant spectral type range, a significantly lower fraction compared with the $52 \%$ corresponding to the morphology-only based classifications. More importantly, the distribution for the "normal," i.e., non- $z$, dwarfs does not show that deficiency of objects with intermediate spectral types 07-07.5 observed in the histograms in Figure 1. Among the Vz stars, however, there is still a predominance of intermediate-type objects. Specifically, $61 \mathrm{O} \mathrm{Vz}$ objects, $\sim 78 \%$ of the whole sample, show spectral types between $\mathrm{O} 6.5$ and O8. As evident from the bottom panel of Figure 8, the populations of $\mathrm{O} \mathrm{Vz}$ and $\mathrm{OV}$ non- $z$ stars are comparable in number at the former subtypes.

We would like to compare the newly obtained spectral type distribution with that for the O V stars in 30 Doradus (Figure 1 of SS14). However, the two Vz samples were selected following different criteria, as the restrictive quantitative condition $z \geqslant 1.10$ was incorporated in this paper for the first time. A detailed comparison will make sense only after the classification methodology is standardized. In any case, both distributions share overall characteristics such as, for example, the mentioned predominance of intermediate types among the Vz stars. Using FASTWIND model predictions, SS14 show that part of the properties of the $\mathrm{O} \mathrm{Vz}$ and $\mathrm{OV}$ stars in 30 Doradus can be explained by a natural combination of stellar parameters. 

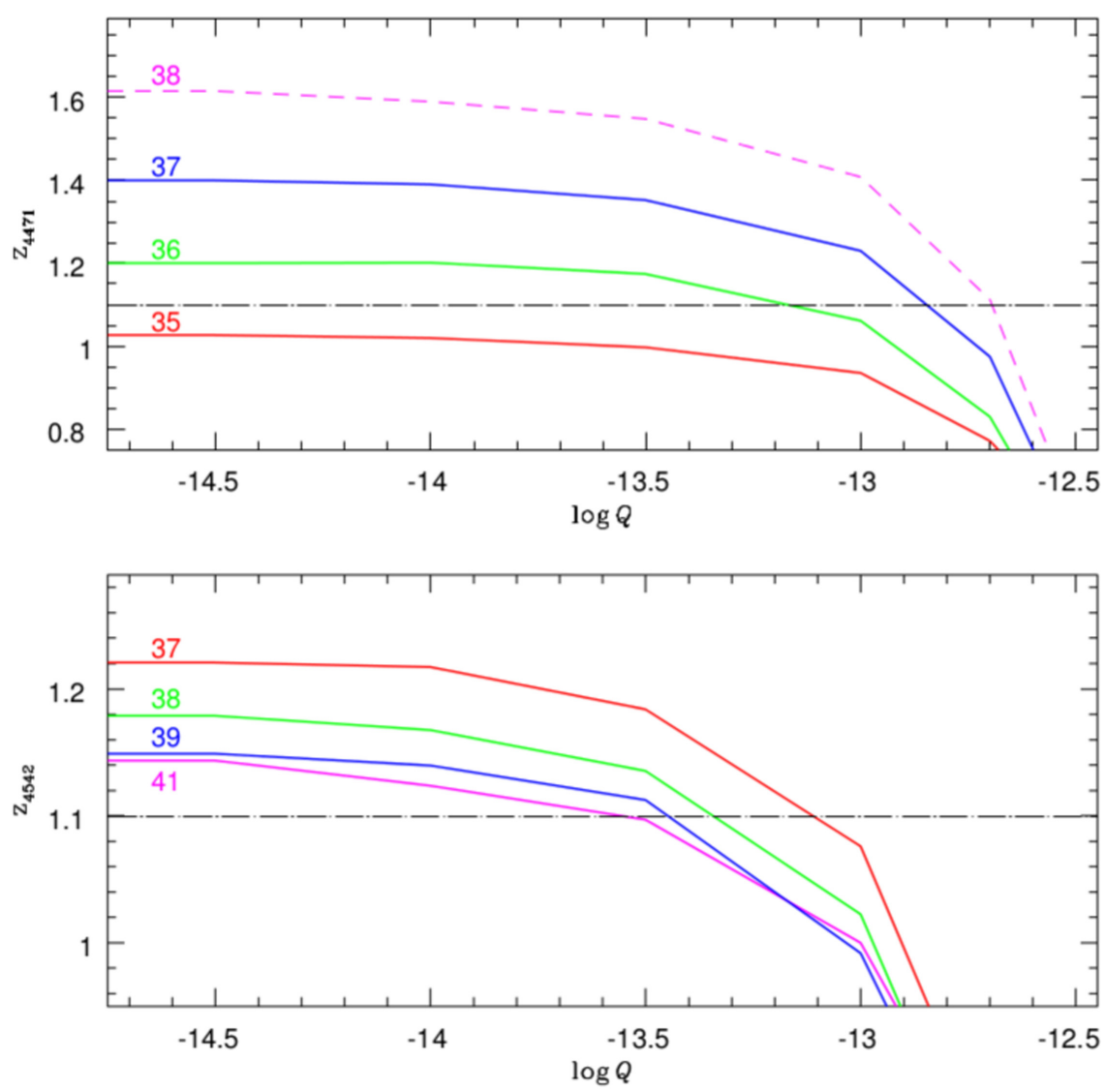

Figure 9. FASTWIND predictions of the behavior of the EW ratios that define the $z$ spectral characteristic, with the variation of the wind-strength parameter $Q$. Fixed values of the resolution $(R=2500)$, surface gravity ( $\log g=4.0 \mathrm{dex})$, and projected rotational velocity $\left(v \sin i=100 \mathrm{~km} \mathrm{~s}^{-1}\right)$ have been considered. The labels next to each color curve indicate the corresponding effective temperature expressed in $\mathrm{kK}$. In the upper panel, the dashed line for $38 \mathrm{kK}$ indicates that this temperature is likely out of the $z_{4471}$ relevant range.

In Section 6, we carry out a similar procedure to investigate the occurrence of the $\mathrm{Vz}$ phenomenon (defined not from the CDs but from EWs of the $\mathrm{He}$ lines) in a solar-metallicity environment. We will show that the distributions in Figure 8 are in good agreement with what it is predicted by the models.

\section{FASTWIND MODEL RESULTS}

In a manner very similar to that followed by SS14, we use synthetic spectra computed with the FASTwIND stellar atmosphere code to investigate how the variation of the most relevant parameters, namely, wind strength $(Q)$ and effective temperature $\left(T_{\text {eff }}\right)$, impact the occurrence of the Vz peculiarity. The differences from the former analysis are twofold: first, we use a grid of models computed for the solar metallicity $Z=Z \odot$, which is an appropriate mean value for the GOSSS stars; second, we consider the quantitative criterion based on the EWs of the $\mathrm{He}_{\mathrm{I}} \lambda 4471$, He II $\lambda 4542$, and $\mathrm{He}$ II $\lambda 4686$ lines (see Section 4.1) for the definition of the $\mathrm{Vz}$ characteristic. The main conclusions from the present analysis are qualitatively the same found by SS14, who were the first to point out the importance of taking into account additional parameters to interpret correctly the $\mathrm{Vz}$ phenomenon. However, numerical results are somewhat different, most probably due to the distinct values of the metallicity considered in each case.

As discussed in Section 4.2, an $\mathrm{O} 7$ dwarf will be classified as $\mathrm{Vz}$ when both $z_{4542}$ and $z_{4471}$ are greater or equal than 1.10 . The effective temperature for this boundary type is represented by $T_{2}$ in Figure 3 . For the values of projected rotational velocity, surface gravity, resolution, and wind strength indicated at the top of that figure, $T_{2}$ is between 36 and 37 $\mathrm{kK}$. Although it depends on the specific values considered for the former parameters, such a value is a reasonable estimate for the limit between the use of $z_{4542}$ and $z_{4471}$.

We first study how $z_{4542}$ and $z_{4471}$ behave with the variation of the wind-strength $\mathrm{Q}$ parameter. Figure 9 shows these parameters as a function of $\log Q$ for a set of different values of the $T_{\text {eff }}$, ranging from 35 to $38 \mathrm{kK}$ for $z_{4471}$ (top panel), and from 37 to $41 \mathrm{kK}$ for $z_{4542}$ (bottom panel). Resolution, surface gravity, and projected rotational velocity are fixed for simplicity. In both panels, the dashed-dotted line at $z=1.10$ marks the value upon which the star is classified as Vz.

The first conclusion that arises from the top panel of Figure 9 is that $\mathrm{O}$ spectra with effective temperatures below a certain value (which is close to $35 \mathrm{kK}$ ) will never be classified as $\mathrm{Vz}$. No matter how weak the stellar wind is, the $z$ parameter for the latest-type stars never exceeds the adopted threshold. This conclusively justifies the decision to exclude all of the dwarfs with spectral types later than O8.5 from the very beginning of the study (Section 3).

Figure 9 also shows that at higher temperatures, the stars may show the $\mathrm{Vz}$ characteristic until the $Q$ parameter takes a certain value denoted as $Q_{\text {lim. }}$. This limit value of $Q$ would represent the strength of the wind required to sufficiently fill in the $\mathrm{He}$ II $\lambda 4686$ absorption, so that the condition $z>1.10$ is broken and, as already shown by SS14, is highly dependent on 
Table 4

Clusters and $\mathrm{H}$ II Regions Containing O Vz Stars

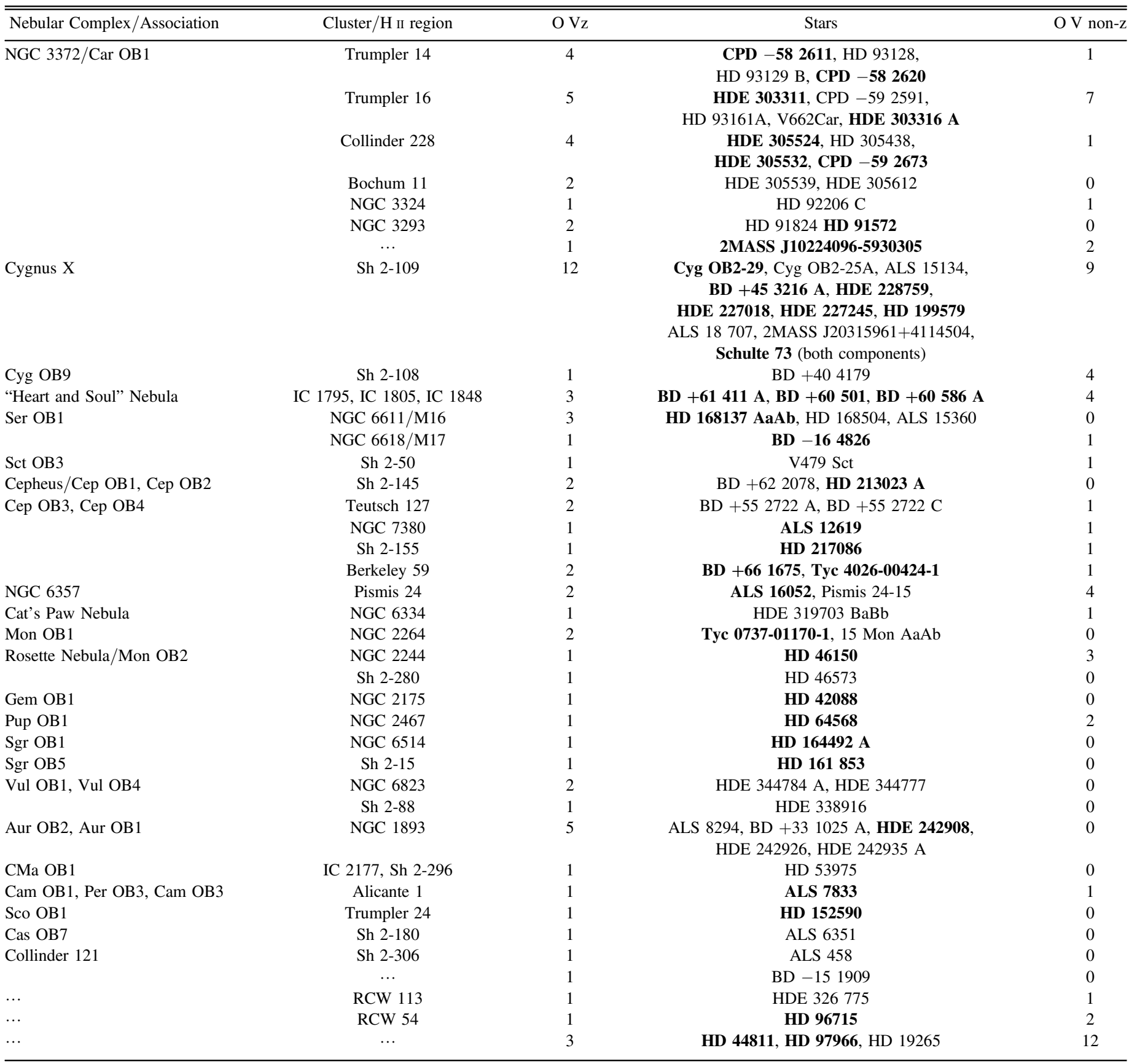

$T_{\text {eff }}$. In a solar-metallicity environment, $Q_{\text {lim }}$ takes its largest value at $37 \mathrm{kK}$, considering either $z_{4542}$ or $z_{4471}$ (in the upper panel, the curve for $38 \mathrm{kK}$ is included for reference, but this temperature is very likely out of the $z_{4471}$ relevant range). Therefore, the main-sequence stars whose $T_{\text {eff }}$ are close to 37 $\mathrm{kK}$ are those that need the strongest stellar winds to no longer show the Vz peculiarity in their spectra. This may explain the predominance of intermediate spectral types in the histogram of Figure 8.

The bottom panel of Figure 9 shows that for very early objects with $T_{\text {eff }}$ above $\approx 39 \mathrm{kK}$, the $\mathrm{Vz}$ characteristic may vanish long before the development of a strong wind; in other words, these stars could be classified as non- $z$ even if they have stellar winds with a strength comparable to other $\mathrm{O} \mathrm{Vz}$ at later types. This naturally explains the observed drop in the number of $\mathrm{O} \mathrm{Vz} \mathrm{stars} \mathrm{for} \mathrm{spectral} \mathrm{types} \mathrm{earlier} \mathrm{than} \mathrm{O6.}$

In the present model configuration, the approximate values of $\log Q_{\mathrm{lim}}$ are the following: -12.85 for $T_{\mathrm{eff}} \approx 37 \mathrm{kK}$ (or -13.1 , if considered $z_{4542}$ ), -13.20 for $T_{\text {eff }} \approx 36 \mathrm{kK}$, and lower than -13.35 for $T_{\text {eff }}$ of the order of and above $38 \mathrm{kK}$. We recall that in this model, the resolution, surface gravity, and projected rotational velocity are fixed for didactic purposes $(R=2500$, $\log g=4.0$, and $v \sin i=100$ ). Although the overall behavior of the $z$ parameter is similar, the limit values of $Q$ can logically vary when alternative configurations are considered, i.e., for different values of $R, \log g$, and $v \sin i$, which is a fortunate fact as the existence of $\mathrm{Vz}$ stars with $T_{\text {eff }}>39 \mathrm{kK}$ would be otherwise very difficult to explain. Anyway, $Q_{\lim }$ for $T_{\text {eff }} \approx$ 
Table 5

Clusters Containing O V Stars but Not Vz Objects

\begin{tabular}{|c|c|c|c|}
\hline $\begin{array}{l}\text { Nebular Complex/ } \\
\text { Association }\end{array}$ & $\begin{array}{l}\text { Cluster/H II } \\
\text { region }\end{array}$ & $\mathrm{OV}$ & Stars \\
\hline$\cdots$ & Pismis 11 & 1 & CPD -49 2322 \\
\hline$\cdots$ & Bochum 1 & 1 & HDE $256725 \mathrm{~A}$ \\
\hline Car OB1 & Bochum 9 & 1 & HD 91837 \\
\hline Sgr OB1 & Collinder 367 & 1 & HD 165921 \\
\hline$\cdots$ & $\begin{array}{l}\text { Havlen-Mof- } \\
\text { fat } 1\end{array}$ & 2 & ALS 18770, ALS 18768 \\
\hline$\cdots$ & IC 1590 & 2 & HD 5005 A, HD 5005 C \\
\hline Cru OB1 & IC 2944 & 3 & $\begin{array}{l}\text { HD 101191, HD 101223, } \\
\text { HD } 101413 \text { AB }\end{array}$ \\
\hline Sgr OB1 & IC 4701 & 1 & HD 167633 \\
\hline$\cdots$ & NGC 6383 & 1 & HD 159176 \\
\hline Ser OB2 & NGC 6604 & 2 & ALS 4880, HD 168461 \\
\hline$\cdots$ & RCW 114 & 1 & HD 155913 \\
\hline Ser OB1 & RCW 161 & 1 & ALS 4923 \\
\hline$\ldots$ & RCW 167 & 1 & BD -10 4682 \\
\hline$\cdots$ & Sh $2-10$ & 1 & Тус 7370-00460-1 \\
\hline Cas OB7, Cas OB1 & Sh $2-185$ & 1 & $\mathrm{BD}+60134$ \\
\hline Cas OB 6 & $\cdots$ & 1 & $\mathrm{BD}+62424$ \\
\hline Aur OB1 & Sh 2-227 & 1 & ALS 8272 \\
\hline Aur OB2, Aur OB1 & Sh $2-230$ & 1 & HD 35619 \\
\hline$\cdots$ & Sh 2-289 & 1 & LS 85 \\
\hline Ser OB1 & Sh $1-48$ & 1 & BD -14 5014 \\
\hline Сер OB2 & Trumpler 37 & 1 & HD $206267 \mathrm{AaAb}$ \\
\hline Vel OB1, Vel OB2 & $\cdots$ & 1 & V467 Vel \\
\hline Cas OB5 & $\cdots$ & 1 & $\mathrm{BD}+602635$ \\
\hline$\ldots$ & $\ldots$ & 1 & $\mathrm{BD}+552840$ \\
\hline Cep OB2 & $\cdots$ & 1 & ALS 12050 \\
\hline Vul OB1, Vul OB4 & $\cdots$ & 1 & HDE 344758 \\
\hline Cen OB1 & $\cdots$ & 1 & HD 110360 \\
\hline
\end{tabular}

$37 \mathrm{kK}$ always appears to be significantly larger than those computed for other effective temperatures. In conclusion, we should note that in a solar-metallicity environment, the O-type stars with effective temperatures close to that value are by far the most "favored by nature" to show the Vz characteristic in their spectra. This fact is in complete agreement with the observed statistics.

We would like to emphasize that the goal of the very simple theoretical analysis performed here is to contribute to our understanding of observations, in particular, the behavior of the measured EWs, as well as the spectral type distribution observed for the Galactic $\mathrm{O} \mathrm{Vz}$ and $\mathrm{O}$ V stars. Thus, we show that FASTWIND model predictions can nicely account for some of the observed characteristics. These results neither contradict nor confirm the hypothesis of the extreme youth of the Vz stars, but only provide evidence, as already done by SS14, for the importance of considering several stellar parameters related to different physical processes when interpretating the $\mathrm{Vz}$ phenomenon.

\section{LOCATIONS OF THE VZ STARS}

When investigating a peculiar or new class of stars, it is important to also consider their environments and companions, which may provide clues to their nature. The initial hypothesis regarding the $\mathrm{Vz}$ class was that it might be younger than the typical class V, based upon the "inverse Of" interpretation of the phenomenon as explained in the Introduction. Thus, we discuss the locations of the Vz stars in this section, with special attention to the most extreme members of the class. In an attempt to quantify the definition of an "extreme" Vz star, we will consider the condition $z \geqslant 1.20$. Although somewhat arbitrary, this limit is useful since it ensures the membership to the $\mathrm{Vz}$ class, even in those cases where measurement errors are large and, at the same time, focus the discussion on the most particular objects, such as, for example, those shown in Figure 7 , for which the depth of the He II $\lambda 4686$ absorption relative to the other $\mathrm{He}$ lines is quite striking.

Table 4 lists the clusters and $\mathrm{H}_{\text {II }}$ regions containing $\mathrm{Vz}$ objects from our current sample of 197 luminosity class V, i.e dwarfs, stars. The number of $z$ dwarfs within each cluster is quoted in the third column of the table. The number of non- $z$ dwarfs is also provided for comparison in the last column. Of course, these numbers are lower limits not only because of observational incompleteness, but also due to the selection criteria for the measurable sample (Section 3). Column 4 details the names of the $z$ dwarfs in each cluster. The 39 "extreme" members, as defined previously, are marked in bold. For comparison and control, Table 5 provides an analogous listing for the clusters that contain normal, i.e., non- $z$, dwarfs but do not include $\mathrm{Vz}$ objects.

Table 4 contains $52 \mathrm{Vz}$ stars in 15 clusters and/or $\mathrm{HII}$ regions that contain two or more $\mathrm{Vz}$ objects, all of which have ages less than about $3 \mathrm{Ma}$ as far as is known; at greater cluster ages, $\mathrm{H}_{\text {II }}$ regions are generally absent (Walborn 2010). The same Table also includes 23 single (to date) Vz stars in 23 young clusters and/or $\mathrm{H}$ II regions, for a grand total of 75 found in such environments. Only three Vz objects (HD 19265, HD 44811, and HD 97966) cannot be directly associated with a very young cluster. The especially outstanding entries in Table 4 are for the Carina Nebula, with a total of $19 \mathrm{Vz}$ members, half of which are extreme cases, and the extended Cygnus region with 12 , distributed among various subregions in both cases. The $\mathrm{Vz}$ content of the IC 1795/1805/1848 complex, and other clusters such as NGC 6611 and NGC 1893, is also notable. These are all very young objects, providing support for that interpretation of the $\mathrm{Vz}$ class.

Besides the large absolute number of $\mathrm{Vz}$ stars in Carina and Cygnus, which might be expected since those are the two bestrepresented regions in GOSSS, the proportion of $\mathrm{Vz}$ to normal class V objects $(\sim 60 \%)$ is evident. Within the Carina Nebula, the difference between Trumpler 14 and Trumpler 16 is particularly curious. While the former shows a majority of $z$ objects, in the latter there is a predominance of non- $z$ dwarfs. Some authors have suggested an extremely young age of around 0.5 Ma for Trumpler 14 (e.g., Penny et al. 1993; Rochau et al. 2011). On the other hand, although also very young, Trumpler 16 appears to be somewhat older (e.g., Wolk et al. 2011), with evolved supergiants such as the remarkable Luminous Blue Variable $\eta$ Car.

Among the clusters with no $\mathrm{Vz}$ objects (Table 5), we emphasize IC 2944, which contains at least three normal dwarfs (five if the binaries HD 101131 AB and HD 101436 are also taken into account), and shows a tenuous $\mathrm{H}$ II region. McSwain \& Gies (2005) evaluated the age of IC 2944 to be 6.6 Ma. Other salient cases are Havlen-Moffat 1, an evolved cluster containing Wolf-Rayet stars (Havlen \& Moffat 1977), and also IC 1590 and NGC 6604, whose average ages are found to be larger than 6.0 Ma (Kharchenko et al. 2013). All of the clusters from Table 5 are, on average, older than those containing a conspicuous population of $\mathrm{OVz}$ objects, as suggested by the different morphologies observed in the 

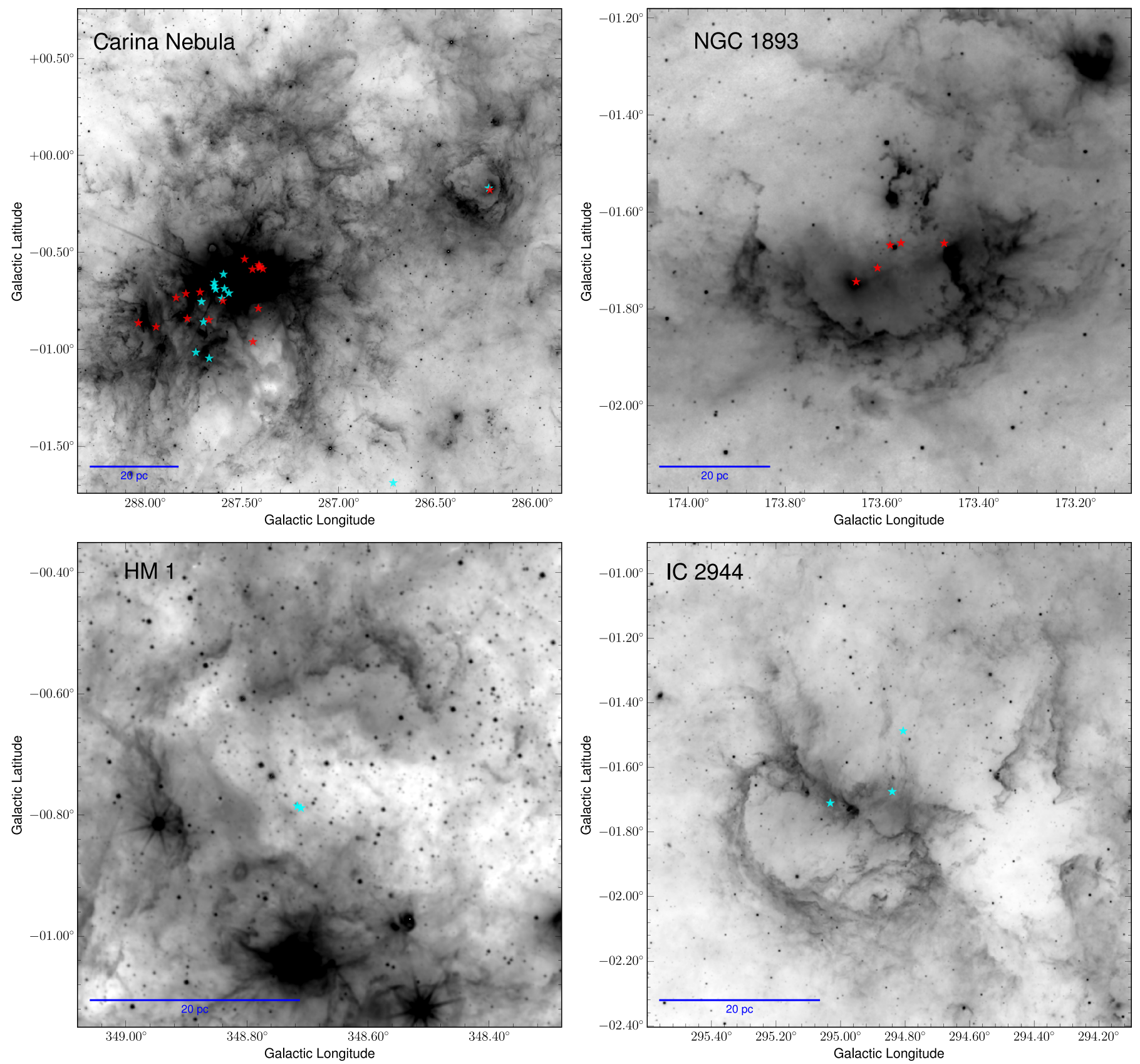

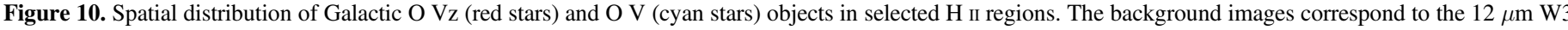

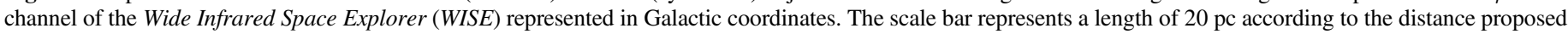
for each region. Top: Carina region (left) and NGC 1893 (right). Bottom: Havlen-Moffat 1 (left) and IC 2944 (right).

mid-infrared images from the Wide-field Infrared Survey Explorer (WISE) (Figure 10). The change in the morphology of a massive star-forming region with the evolutionary stage of the underlying massive stellar content is nicely presented by Koenig et al. (2012). Using the WISE images in the W3 channel $(12 \mu \mathrm{m})$, these authors show the correlation between the age of the main exciting stellar cluster and the structure of the diffuse emission nebulosity produced by excited polycyclic aromatic hydrocarbon molecules (PAH) and small dust grains. Younger massive stellar clusters lack a cleared-out cavity around the powering $\mathrm{O}$ stars. Within a timescale of 3-5 Ma, the $\mathrm{H}$ II regions are cleared out and the observed $\mathrm{PAH}$ and dust emission is mostly concentrated in peripheric ring structures with protuberances or pillars where a new, triggered generation of stars is forming. This evolutive scenario of massive star formation was previously presented by Walborn \& Parker (1992; see also Walborn et al. 1999) and named "two-stage starbursts" scenario.

Finally, in Figure 11, we present the spatial distribution of the Galactic $z$ (red circles) and non- $z$ (cyan circles) O dwarfs, overplotted on the $\mathrm{H} \alpha$ all-sky map produced by Finkbeiner (2004). The Vz objects, especially the most extreme objects, seem to show a higher concentration toward the most active star-forming regions. Of course, normal dwarfs are also found within these regions but, as a class, the latter appear to be more disseminated within and outside of the Galactic disk. In conclusion, we can note that while further astrophysical investigation is needed, the extreme Vz objects may reasonably 


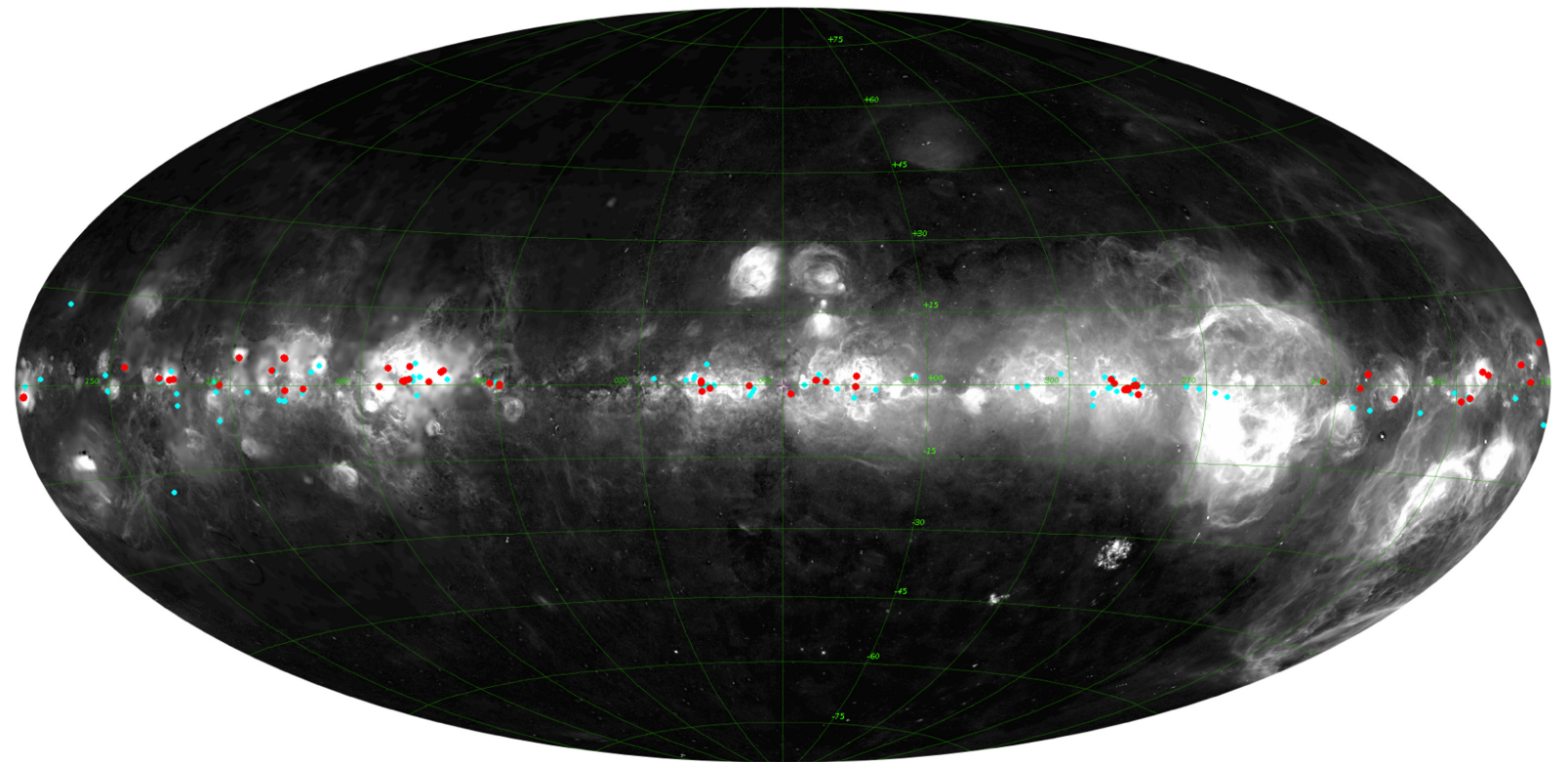

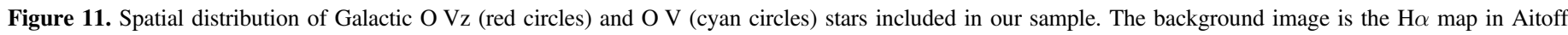
projection produced by Finkbeiner (2004) and represented in Galactic coordinates.

be hypothesized to represent the youngest, post-embedded massive stars.

\section{SUMMARY AND OUTLOOK}

Comprising the largest collection of O-star optical spectra ever assembled, The Galactic O Star Spectroscopic Survey (GOSSS) provides a unique opportunity to investigate in detail and systematically this important class of objects. The $\mathrm{O}$ stars belonging to the Vz luminosity subclass have been suggested to be among the youngest optically observable massive objects, and thus their study appears as key to the understanding of the early evolution of massive stars.

This work has provided new insight into the $\mathrm{Vz}$ spectral classification criteria. The convenience of incorporating a quantitative criterion based on the EWs of the $\mathrm{He}_{\mathrm{I}} \lambda 4471$, He II $\lambda 4542$, and He II $\lambda 4686$ spectral lines was shown. The EWs for a large sample of stars in GOSC were measured, allowing for the recalibration of the $\mathrm{Vz}$ phenomenon. As a result, the threshold of the " $z$ parameter," defined as $z=\mathrm{EW}$ $(\mathrm{He}$ II $\lambda 4686) / \operatorname{Max}\left[\mathrm{EW}\left(\mathrm{He}_{\mathrm{I}} \lambda 4471\right), \quad \mathrm{EW}(\mathrm{He}\right.$ II $\left.\lambda 4542)\right], \quad$ upon which a spectrum is classified as $\mathrm{Vz}$, was increased to 1.10 . This change is made to avoid marginal cases, whose actual membership in the $\mathrm{Vz}$ class is unclear and therefore may be contributing to the recent controversy that has arisen regarding the relation between the $\mathrm{Vz}$ classification and the small age of the objects. The new critical value for the $z$ parameter proposed in this work was adopted in the third installment of the GOSSS project. This led to a complete revision of the spectral types in GOSC and, more importantly, to the definition for the first time of $\mathrm{O} \mathrm{Vz}$ standard stars.

The population of $\mathrm{O} \mathrm{V}$ and $\mathrm{O} \mathrm{Vz}$ stars resulting from the new classification criteria were comparatively analyzed. With 78 members, the $\mathrm{O} \mathrm{Vz}$ objects represent $\sim 40 \%$ of the dwarfs in the relevant spectral range of $\mathrm{O} 3-\mathrm{O} 8.5$. However, their distribution as a function of spectral type is not uniform but shows a marked concentration toward intermediate types. Based on a very simple theoretical analysis performed using synthetic spectra from a grid of FASTWIND models computed for a solar-metallicity value, we showed that the former, as well as other observed characteristics in the spectral type distribution of the $\mathrm{OVz}$ stars, are in perfect agreement with what is predicted by the models. This study confirmed the result from SS14 on the complexity, previously unrecognized, of the $\mathrm{Vz}$ phenomenon and the necessity of taking into account many processes for its correct interpretation.

The locations, environment, and companions of the $\mathrm{O} \mathrm{Vz}$ stars were analyzed, with special attention given to the most extreme members. Almost all of the $\mathrm{OVz}$ objects are associated with very young clusters, all of which have ages less than $3 \mathrm{Ma}$ as far as we know. On the other hand, clusters with no $\mathrm{Vz}$ objects are clearly older, as evidenced by the morphologies observed in the mid-infrared WISE images and the presence of more evolved stars. These facts add considerable support to the interpretation of the $\mathrm{Vz}$ characteristic as a signature of youth, although further astrophysical investigation is needed.

The results presented here represent the first steps toward our goal of understanding the physical significance of the $\mathrm{O} \mathrm{Vz}$ stars. We have defined a robust sample of O Vz stars, many of which are presumably single and suitable to perform quantitative spectroscopic analyses in high resolution which will allow us to derive their stellar and wind parameters. Such a study, which will be presented in a forthcoming paper, will certainly establish the actual evolutionary status of these interesting objects.

We thank the anonymous referee for useful suggestions and comments on the manuscript. We also thank the staff of Las Campanas Observatory for their support during the observing runs. J.I.A. and R.H.B. acknowledge financial support from the Chilean Government grants, through the FONDECYT Iniciación 11121550 and FONDECYT Regular 1140076 projects, respectively. S.S.-D. acknowledges financial support from the Spanish Ministry of Economy and Competitiveness (MINECO) under grants AYA2010-21697-C05-04, and Severo Ochoa SEV-2011-0187, and by the Canary Islands Government under 
grant PID2010119. J.M.A., A.S., and E.J.A. acknowledge support from [a] the Spanish Government Ministerio de Economía y Competitividad (MINECO) through grants AYA2010-15 081, AYA2010-17 631, and AYA2013-40 611$\mathrm{P}$ and $[\mathrm{b}]$ the Consejería de Educación of the Junta de Andalucía through grant P08-TIC-4075. C.S.-S. acknowledges support from the Joint Committee ESO-Government of Chile. I.N. and A.M. acknowledge support from the Spanish Ministerio de Ciencia e Innovación (MICINN) under grant AYA2012-39364-C02-02, and by the Generalitat Valenciana (ACOMP/2014/129). A.M. acknowledges support from the Generalitat Valenciana through the grant BEST/2015/242. The Space Telescope Science Institute is operated by the Association of Universities for Research in Astronomy, Inc., under NASA contract NAS5-26555. This research has made use of Aladin (Bonnarel et al. 2000), and the SIMBAD database, operated at CDS, Strasbourg, France.

\section{REFERENCES}

Barbá, R. H., Gamen, R. C., Arias, J. I., et al. 2010, RMxAC, 38, 30 Bonnarel, F., Fernique, P., Bienaymé, O., et al. 2000, A\&A, 143, 33

Conti, P. S., \& Alschuler, W. R. 1971, ApJ, 170, 3

Conti, P. S., \& Leep, E. M. 1974, ApJ, 193, 113

Evans, C. J., Taylor, W. D., Hénault-Brunet, V., et al. 2011, A\&A, 530, A108 Finkbeiner, D. P. 2004, ApJ, 614, 186

Havlen, R. J., \& Moffat, A. F. J. 1977, A\&A, 58, 351

Kharchenko, N. V., Piskunov, A. E., Schilbach, E., et al. 2013, A\&A, 558A, 53 Koenig, X. P., Leisawitz, D. T., Benford, D. J., et al. 2012, ApJ, 744, 130

Lorenzo, J., Simón-Díaz, S., Negueruela, I., \& Vilardell, F. 2010, in ASP Conf. Ser. 435, Binaries Key to Comprehension of the universe, ed. A. Prsa, \& M. Zejda (San Francisco, CA: ASP), 409

Maíz Apellániz, J., Alfaro, E. J., Arias, J. I., et al. 2015, in Proc. of the XI Scientific Meeting of the Spanish Astronomical Society, Highlights of Spanish Astrophysics VIII, ed. A. J. Cenarro et al., 603

Maíz Apellániz, J., Pellerin, A., Barbá, R. H., et al. 2012, in ASP Conf. Ser., Vol. 465, Proc. of a Scientific Meeting in Honor of Anthony F. Moffat held at Auberge du Lac Taureau (San Francisco, CA: ASP), 484

Maíz Apellániz, J., Sota, A., Arias, J. I., et al. 2016, ApJS, 224, 4

Maíz Apellániz, J., Sota, A., Walborn, N. R., et al. 2011, in Proc. IX Scientific Meeting of the Spanish Astronomical Society (SEA), Highlights of Spanish Astrophysics VI, ed. M. R. Zapatero Osorio et al., 467
Maíz Apellániz, J., Walborn, N. R., Galué, H. A., et al. 2004, ApJS, 151, 103 Markova, N., Puls, J., Scuderi, S., et al. 2011, A\&A, 530, 11

Martins, F., Schaerer, D., \& Hillier, D. J. 2005, A\&A, 436, 1049

Mason, B. D., Hartkopf, W. I., Gies, D. R., et al. 2009, AJ, 137, 3358

Mathys, G. 1988, A\&AS, 76, 427

Mathys, G. 1989, A\&AS, 81, 237

McSwain, M. V., \& Gies, D. R. 2005, ApJS, 161, 118

Parker, J. W., Garmany, C. D., Massey, P., \& Walborn, N. R. 1992, AJ, 103, 1205

Penny, L. R., Gies, D. R., Hartkopf, W. I., et al. 1993, PASP, 105, 588

Puls, J., Urbaneja, M. A., Venero, R., et al. 2005, A\&A, 435, 669

Rochau, B., Brandner, W., Stolte, A., et al. 2011, MNRAS, 418, 949

Sabín-Sanjulián, C., Simón-Díaz, S., Herrero, A., et al. 2014, A\&A, 564, A39

Sana, H., de Koter, A., de Mink, S. E., et al. 2013, A\&A, 550A, 107

Sana, H., Le Bouquin, J.-B., Lacour, S., et al. 2014, ApJS, 215, 15

Santolaya-Rey, A. E., Puls, J., \& Herrero, A. 1997, A\&A, 323, 488

Simón-Díaz, S., Castro, N., García, M., et al. 2011a, in Proc. IAU Symp. 272, Active OB Stars: Structure, Evolution, Mass Loss, and Critical Limits, ed. C. Neiner et al. (Cambridge: Cambridge Univ. Press), 310

Simón-Díaz, S., Castro, N., Herrero, A., et al. 2011b, JPhCS, 328, 012021

Simón-Díaz, S., Herrero, A., Sabín-Sanjulián, C., et al. 2014, A\&A, 570L, 6

Simón-Díaz, S., Negueruela, I., Maíz Apellániz, J., et al. 2015, in Proc. of the XI Scientific Meeting of the Spanish Astronomical Society, Highlights of Spanish Astrophysics VIII, ed. A. J. Cenarro et al., 576

Sota, A., Maíz Apellániz, J., Morrell, N. I., et al. 2014, ApJS, 211, 10

Sota, A., Maíz Apellániz, J., Walborn, N. R., et al. 2011, ApJS, 193, 24

Tokovinin, A., Mason, B. D., \& Hartkopf, W. I. 2010, AJ, 139, 743

Walborn, N. R. 1973, ApJ, 179, 517

Walborn, N. R. 2001, in ASP Conf. 242, Eta Carinae and Other Mysterious Stars: The Hidden Opportunities of Emission Spectroscopy, ed. T. R. Gull, S. Johannson, \& K. Davidson (San Francisco, CA: ASP), 217

Walborn, N. R. 2009, in STScI Symp. Ser. No. 20, Massive Stars: From Pop III and GRBs to the Milky Way, ed. M. Livio, \& E. Villaver (Cambridge: Cambridge Univ. Press), 167

Walborn, N. R. 2010, in ASP Conf. 425, Hot and Cool: Bridging Gaps in Massive Star Evolution, ed. C. Lietherer, P. Bennett, P. Morris, \& J. van Loon (San Francisco, CA: ASP), 45

Walborn, N. R., Barbá, R. H., Brandner, W., et al. 1999, AJ, 117, 225

Walborn, N. R., \& Blades, J. C. 1997, ApJS, 112, 457

Walborn, N. R., \& Fitzpatrick, E. L. 1990, PASP, 102, 379

Walborn, N. R., Maíz Apellániz, J., Sota, A., et al. 2011, AJ, 142, 150

Walborn, N. R., \& Parker, J. W. 1992, ApJ, 399L, 87

Walborn, N. R., Sana, H., Simón-Díaz, S., et al. 2014, A\&A, 564, A40

Walborn, N. R., Sota, A., Maíz Apellániz, J., et al. 2010, ApJL, 711, L143

Wolk, S. J., Broos, P. S., Getman, K. V., et al. 2011, ApJS, 194, 12 